\title{
Spatiotemporal analysis of endocytosis and membrane distribution of fluorescent sterols in living cells
}

\author{
Daniel Wüstner · Nils J. Færgeman
}

Accepted: 30 July 2008/ Published online: 12 September 2008

(C) The Author(s) 2008. This article is published with open access at Springerlink.com

\begin{abstract}
Distribution and dynamics of cholesterol in the plasma membrane as well as internalization pathways for sterol from the cell surface are of great cell biological interest. Here, UV-sensitive wide field microscopy of the intrinsically fluorescent sterols, dehydroergosterol (DHE) and cholestatrienol (CTL) combined with advanced image analysis were used to study spatiotemporal sterol distribution in living macrophages, adipocytes and fibroblasts. Sterol endocytosis was directly visualized by time-lapse imaging and noise-robust tracking revealing confined motion of DHE containing vesicles in close proximity to the cell membrane. Spatial surface intensity patterns of DHE as well as that of the lipid marker DiIC12 being assessed by statistical image analysis persisted over several minutes in cells having a constant overall curvature. Sites of sterol endocytosis appeared indistinguishable from other regions of the cell surface, and endocytosis contributed by $62 \%$ to total sterol uptake in J774 cells. DHE co-localized with fluorescent transferrin (Tf) in vesicles right after onset of endocytosis and in deepened surface patches of energy depleted cells. Surface caveolae labeled with GFP-tagged caveolin were not particularly enriched in DHE or CTL. Some sterol co-localized with internalized caveolin suggesting that caveolar endocytosis contributes to vesicular sterol uptake. These findings demonstrate that plasma membrane sterol is internalized by several endocytic
\end{abstract}

Electronic supplementary material The online version of this article (doi:10.1007/s00418-008-0488-6) contains supplementary material, which is available to authorized users.

D. Wüstner $(\bowtie) \cdot$ N. J. Færgeman

Department of Biochemistry and Molecular Biology,

University of Southern Denmark, Campusvej 55,

5230 Odense M, Denmark

e-mail: wuestner@bmb.sdu.dk pathways. Sterol endocytosis does not require formation of microscopically resolvable sterol clusters or enrichment of sterol in surface caveolae.

Keywords Cholesterol $\cdot$ Membrane . Fluorescence . Time-lapse · Tracking · Wavelet · Fourier · Caveolin
Abbreviation
Alexa546-Tf Alexa546-tagged transferrin
CTL Cholestatrienol
DHE Dehydroergosterol
GFP Green fluorescent protein
ERC Endocytic recycling compartment
MCD Methyl- $\beta$-cyclodextrin
MSD Mean square displacement

\section{Introduction}

Cholesterol has a tightly controlled intracellular distribution with the highest concentration in the plasma membrane. Previous studies have shown that cholesterol and related sterols move by vesicular and non-vesicular pathways between the plasma membrane and intracellular compartments (Baumann et al. 2005; Hao et al. 2002; Kaplan and Simoni 1985; Li and Prinz 2004; Wüstner et al. 2002). Employing the intrinsically fluorescent sterol dehydroergosterol (DHE), it was shown that the endocytic recycling compartment (ERC) is a major sterol storage compartment under basal conditions in non-polarized cells including macrophages (Hao et al. 2002; Wüstner et al. 2005). This could be verified by co-labeling with the recycling marker transferrin (Tf). Using DHE, it was demonstrated that there is a high non-vesicular sterol flux 
from the plasma membrane to the ERC in Chinese hamster ovarian (CHO) cells, while recycling of DHE back to the cell surface occured in vesicles and required key regulators of recycling like RME-1 (Hao et al. 2002; Lin et al. 2001). In contrast, in polarized hepatic HepG2 cells sterol transport to the subapical compartment/apical recycling compartment (SAC/ARC) required ATP and occurred in vesicles partially containing Tf (Wüstner et al. 2002). Thus, vesicular and non-vesicular pathways contribute to sterol uptake from the plasma membrane; though sterol endocytosis was not yet directly observed by fluorescence microscopy.

There is currently an ongoing debate about whether cholesterol can phase segregate into sterol-rich and -poor domains in the plasma membrane; a central statement of the raft hypothesis (Simons and Ikonen 1997; Zhang et al. 2005). Using multiphoton microscopy, Schroeder and colleagues analyzed staining patterns of DHE in the cell membrane of fibroblasts (Zhang et al. 2005). The obtained results were interpreted as supporting evidence for the presence of sterol domains in the plasma membrane of living cells. Recent experiments using high sensitivity wide field UV microscopy of various cell types being exclusively labeled with DHE in the plasma membrane confirm a non-homogeneous distribution of DHE in mammalian cell membranes (Wüstner 2007b). Multicolor fluorescence imaging showed, however, that DHE's surface pattern coincides with that of fluid phase phospholipid probes like short chain diindocarbocyanine (DiI) dyes and BODIPYlabeled phosphatidylcholine (BODIPY-PC) (Wüstner 2007b). By 3D wide field microscopy it was found that DHE staining patterns have typical correlation lengths of $0.8-1.5 \mu \mathrm{m}$ measured over several image planes. DHE showed homogeneous staining of plasma membrane blebs induced by F-actin disruption. These and other results demonstrated that the rough surface topology of mammalian cell membranes but not lateral domains cause a heterogeneous plasma membrane staining of DHE (Wüstner 2007b).

Previous studies provide no temporal information regarding the time evolution of DHE membrane staining patterns and did not try to correlate membrane sterol distribution to sterol internalization. It is, for example, possible that sterol domains form temporally at specific plasma membrane sites during formation of endocytic vesicles. In this study, dynamic plasma membrane staining patterns of DHE were statistically assessed and compared to a membrane lipid probe, diindocarbocyanine (DiIC12). Co-localization analysis revealed that some DHE is internalized together with transferrin (Tf), and that both probes have overlapping distribution at the cell surface.

It is an accepted view that caveolae are particularly enriched in cholesterol, but the sterol concentration in these surface regions remains uncertain (Ikonen 2000; Maxfield and Wüstner 2002). We show that neither DHE nor the related cholestatrienol (CTL) become enriched in cellsurface caveolae visualized with GFP-tagged caveolin in fibroblasts or in caveolar invaginations in adipocytes. Despite the absence of sterol clustering in caveolae, we observed occasionally co-localization of fluorescent sterols with internalized caveolae suggesting that caveolar endocytosis contributes to vesicular sterol uptake.

\section{Materials and methods}

Chemicals and reagents

Medium 1 contained $150 \mathrm{mM} \mathrm{NaCl}, 5 \mathrm{mM} \mathrm{KCl}, 1 \mathrm{mM}$ $\mathrm{CaCl}_{2}, 1 \mathrm{mM} \mathrm{MgCl} 2,5 \mathrm{mM}$ glucose and $20 \mathrm{mM}$ HEPES (pH 7.4). Medium 2 was identical to medium 1 except that it contained no glucose but $5 \mathrm{mM}$ sodium azide and $50 \mathrm{mM}$ 2-deoxyglucose for energy depletion of cells. Fetal calf serum (FCS) and DMEM were from GIBCO BRL. 1,1'-didodecyl-3,3,3',3'-tetramethylindocarbocyanine perchlorate (DiIC12(3)) and succinimidyl esters of Alexa-546 were purchased from Molecular Probes Inc. (Eugene, Oregon, USA). CTL was obtained from Avanti Polar Lipids (Alabaster, USA). All other chemicals were from Sigma Chemical. Transferrin (Tf) was iron loaded as described previously (Yamashiro 1984). Succinimidyl ester of Alexa-488 and Alexa-546 were then conjugated to the iron-loaded Tf following the manufacturer's instructions. The GFP-caveolin 1 (GFP-Cav1) plasmid was a generous gift from Dr. Bo van Deurs, University of Copenhagen, Denmark (Thomsen 2002). DHE and CTL were loaded on methyl-beta-cyclodextrin (DHE(MCD or CTL/MCD) following exactly our previously published procedure (Hao et al. 2002; Wüstner 2007b, 2002).

\section{Cell culture}

Murine macrophage J774 cells and Chinese hamster ovarian (CHO) cells were grown in DMEM with $4.5 \mathrm{~g} / 1$ glucose, supplemented with $10 \%$ heat-inactivated FCS and antibiotics as described (Hao et al. 2002; Wüstner et al. 2005). 3T3-L1 fibroblasts were cultured in DMEM containing $10 \%$ calf serum. Confluent cultures were induced to differentiate 2 days after reaching confluence by the addition of DMEM containing $10 \% \mathrm{FCS}, 1 \mu \mathrm{m} / \mathrm{ml}$ insulin, $115 \mu \mathrm{g} / \mathrm{ml}$ methylisobutylxanthine and $390 \mathrm{ng} / \mathrm{ml}$ dexamethasone. After $48 \mathrm{~h}$, the differentiation medium was replaced by fresh FCS/DMEM containing $1 \mu \mathrm{g} / \mathrm{ml}$ insulin, while after another $48 \mathrm{~h}$, cells were kept in DMEM/FCS only until start of the experiment. For experiments, cells were plated onto glass coverslip coated 
with poly-D-lysine as described previously (Salzmann and Maxfield 1989).

Labeling of J774 cells and adipocytes with fluorescent probes and sterols

J774 cells or 3T3-L1 adipocytes were labeled with DHE/ $\mathrm{M} \beta \mathrm{CD}$ for 5 or $2 \mathrm{~min}$ at $37^{\circ} \mathrm{C}$, respectively. This short labeling pulse was found to be sufficient for achieving high plasma membrane staining. Cells were washed and further incubated at $37^{\circ} \mathrm{C}$ for the indicated time. $\mathrm{J} 774$ cells were incubated for $5 \mathrm{~min}$ at $37^{\circ} \mathrm{C}$ with DHE/M $\beta \mathrm{CD}$ containing $10 \mu \mathrm{g} / \mathrm{ml}$ Alexa 546-Tf, washed, and imaged on a wide field microscope (see below). In some experiments, doublelabeled cells were fixed with $2 \%$ paraformaldehyde (PFA) for $10 \mathrm{~min}$ on ice and washed extensively, allowing for acquisition of image stacks without vesicle movement. For energy depletion, cells were incubated for $30 \mathrm{~min}$ at $37^{\circ} \mathrm{C}$ in Medium 2. Alternatively, cells were ATP-depleted by incubation for $5 \mathrm{~min}$ at $37^{\circ} \mathrm{C}$ with $100 \mathrm{mM}$ sodium azide and $100 \mathrm{mM}$ 2-deoxyglucose as described (Zha 1998). Both methods gave identical results regarding inhibition of receptor-mediated endocytosis, as shown previously (Wüstner et al. 2005). Co-localization of DHE with Alexa546-Tf was determined in ATP-depleted J774 cells as described for control cells, except that all incubations were performed in Medium 2. J774 cells were labeled with DiIC12 made from a stock solution and bound to BSA as described previously (Wüstner 2007b).

Expression of GFP-caveolin and analysis of sterol distribution in caveolae

GFP-Cav1 was transiently expressed in CHO cells using lipofectamine as described by the manufacture (Invitrogen A/S Taastrup, Denmark). 3T3 L1 adipocytes were transiently transfected with GFP-Cav1 by electroporation as described (Zeigerer et al. 2002). Briefly, cells were differentiated as described above and 4 days after beginning this process, cells were electroporated at $180 \mathrm{~V}$ and $950 \mu \mathrm{F}$ with $45 \mu \mathrm{g}$ plasmid DNA and plated onto coverslip-bottom dishes coated with poly-lysine as described above. Transfected adipocytes were used for experiments 24-72 h after transfection. Cells were pulse-labeled with either DHE/MCD or CTL/MCD and directly imaged, or, additionally labeled with DiIC12 as described above. To visualize the caveolae rich surface of adipocytes, 3T3-L1 adipocytes were labeled with DHE/MCD as described above, washed and pulse labeled with DiIC12, placed on ice and labeled with $50 \mu \mathrm{g} / \mathrm{ml}$ Alexa488-CTxB. Cells were washed once with ice-cold and twice with warmed Medium 1 and imaged on a wide field microscope as described below.
Fluorescence microscopy

Wide field fluorescence microscopy and digital image acquisition were carried out using a Leica DMIRB microscope with a $63 \mathrm{x}, 1.4$ NA oil immersion objective (Leica Lasertechnik GmbH, Wetzlar, Germany) equipped with Princeton Instruments cooled CCD camera driven by Image-1/MetaMorph Imaging System software (Universal Imaging Inc., Downington, PA, USA). Alternatively, a Leica DMIRBE microscope containing the same optical components equipped with a Hamamatsu Orca BT512 4-stage peltier and water cooled $\left(-80^{\circ} \mathrm{C}\right) \mathrm{CCD}$ camera (Hamamatsu Photonics Inc., HamamatsuCity, Japan) and a Lambda SC smartshutter (Sutter Instrument Company, Novato, CA, USA), driven by ImagePro Plus and ScopePro (Media Cybernetics, Inc., Silver Spring, MD, USA) was used. Alexa546-Tf and DiIC12 were imaged using a standard rhodamine filter set [535-nm (50 nm bandpass) excitation filter, 565-nm longpass dichromatic filter, and 610-nm $(75 \mathrm{~nm})$ bandpass) emission filter]. Alexa488CTxB's and GFP-Cav1's fluorescence were collected using a standard fluorescein filter set [470-nm, (20 nm bandpass) excitation filter, 510-nm longpass dichromatic filter, and $537-\mathrm{nm}$ (23 nm bandpass) emission filter]. Detection of DHE's and CTL's emission was made possible using a UV adapted wide field microscope with a filter cube obtained from Chroma Technology Corp. (Brattleboro, USA) with $335-\mathrm{nm}$ (20 $\mathrm{nm}$ bandpass) excitation filter, 365-nm longpass dichromatic filter, and 405-nm (40-nm bandpass) emission filter as described previously (Wüstner 2007b). Axial and lateral chromatic aberration of the microscope in the UV compared to the red channel was determined and corrected as described elsewhere (Wüstner and Faergeman 2008). Differential interference contrast (DIC) and bright field imaging were performed using appropriate optics.

Time-lapse imaging was performed on living J774 cells after pulse-labeling with DHE/MCD (see above). After labeling, cells were immediately placed on a temperaturecontrolled stage of the microscope, maintained at $36 \pm 1{ }^{\circ} \mathrm{C}$. Images were acquired every $30 \mathrm{~s}$ for a total of 20 min with only $500 \mathrm{~ms}$ integration time compared to otherwise $2 \mathrm{~s}$ acquisition for minimizing DHE photobleaching. The microscope was placed on a nitrogen-floated table in order to reduce vibrations and thereby to prevent shifts of the focal plane during observation. Time-lapse imaging of DiIC12 labeled cells was performed identically except that acquisition time was much shorter due to the higher quantum yield of the probe.

Image and data analysis

Image analysis was carried out using the software packages Image-1/MetaMorph Imaging System (Universal Imaging 
Inc., Downington, PA, USA), ImagePro Plus (Media Cybernetics, Inc., Silver Spring, MD, USA) or NIH image in form of Scion Image (Scion Corporation, Frederick, Maryland, USA) or ImageJ (developed at the US National Institutes of Health and available on the Internet at http://rsb.info.nih.gov/ij). For presentation purposes and contrast adjustment Adobe Photoshop (Adobe Systems Inc., USA) was used. Time-lapse sequences were first corrected for bleaching of DHE as described (Wüstner 2005). For particle tracking, a plugin to ImageJ developed by Dr. Sage (Swiss Federal Insitute of Technology, Lausanne, Switzerland) was used. This algorithm uses a Mexican hat filter after image alignment to detect Gaussian-like spots in the presence of high noise levels (Sage et al. 2005). Before tracking, image contrast was enhanced using ImageJ tools with $0.0 \%$ pixel saturation over the whole time lapse stacks. Alternatively, a spot enhancing filter implemented in the ImageJ plugin was applied for contrast enhancement of the moving particles. Both techniques gave identical tracking results. The particle trajectory is obtained by a dynamic programming routine which allows for detecting a DHE containing vesicle even in the presence of continuous photobleaching, i.e., diminishing particle intensity. Extensive testing of the algorithm by the authors of this study revealed that trajectories of simulated particles are accurately described for continuously degrading peak signal-to-noise levels (see Fig. 6 in Sage et al. 2005). Thus, the algorithm is very suitable for tracking DHE containing vesicles in living cells. From obtained trajectories, the mean square displacement (MSD) of vesicles was calculated according to:

$$
\begin{aligned}
\operatorname{MSD}(n \cdot \delta t)= & \frac{1}{N-1} \sum_{j=1}^{N-n}\left\{[x(j \cdot \delta t+n \cdot \delta t)-x(j \cdot \delta t)]^{2}\right. \\
& \left.+[y(j \cdot \delta t+n \cdot \delta t)-y(j \cdot \delta t)]^{2}\right\}
\end{aligned}
$$

where $x(t)$ and $y(t)$ are the coordinates of a DHE containing vesicle at time $t, \delta t$ is the time interval at which images were taken, $N$ is the total number of images in a recorded sequence and $n$ and $j$ are positive integers with $n=1,2, \ldots$, $(N-1)$. The obtained MSD curve was fitted to a model of Brownian diffusion confined to an area $A$ by (Daumas 2003; Saxton 1997):

$\operatorname{MSD}(t)=A \cdot(1-\exp (-k \cdot t))+4 \cdot D \cdot t$.

Here, $D$ is the 2-dimensional diffusion coefficient; $k$ is a rate constant describing the confinement process and $t$ is time. An in-house developed FORTRAN program was used to calculate the MSD from particle trajectories using Eq. 1. For image deconvolution and co-localization analysis, a Maximum likelihood restoration method using the Huygens software was applied to z-stacks of DHE/
Alexa546-Tf double labeled cells (Scientific Volume Imaging, Hilversum, The Netherlands). Corresponding single spatially aligned planes of DHE and Alexa546-Tf were background corrected as described (Ghosh et al. 1994). Co-localization was judged either based on line scans through regions of interest (ROI) obtained by ImagePro Plus (Media Cybernetics, Inc., USA) or using the co-localization analyzer module of Huygens software. The latter package was also employed for 3-dimensional surface reconstructions of colocalizing areas along the plasma membrane of ATP-depleted J774 cells.

To determine the plasma membrane staining patterns of DHE, time-lapse data was first corrected for photobleaching of DHE as described (Wüstner 2005). Note that photobleaching of DHE in the plasma membrane following a single exponential decay was spatially invariant, i.e., did not depend on the position as recently shown by bleach rate imaging of DHE (Wüstner 2007b). This was confirmed for the data set used here (not shown). Plasma membrane staining patterns of DHE and DiIC12 were measured from bleaching-corrected time-lapse data using a line scan method along the cell perimeter as described previously with one exception (Wüstner 2007b). The dynamic membrane distribution of DHE and DiIC12 was analyzed after straightening surface segments using a cubic-spline interpolation routine implemented as plugin to ImageJ and written by Dr. Kocsis (Laboratory of Structural Biology, NIAMS, National Institutes of Health, Bethesda MD, USA) (Kocsis et al. 1991). In this routine, the user selects a set of nodes per mouse click; a non-uniform cubic Spline is fitted to these points and the filament is straightened along it. The width of the filament can be defined and was set to 10 pixels in order to cover the whole width of the plasma membrane. Line scans were performed on straightened plasma membrane segments using ImagePro Plus (Media Cybernetics, Inc., Silver Spring, MD, USA). Pixel intensities were exported as ASCII file to SigmaPlot 9.0 (SPSS Inc, Chicago, IL, USA) and correlation in staining patterns were measured using a bivariate analysis routine implemented in this software defining a correlation coefficient $R$ as function of time or plane number for comparing image sequences or stacks, respectively.

$$
R(n)=\frac{\sum\left(\left(x_{i}(n)-\bar{x}(n)\right) \cdot\left(y_{i}(n)-\bar{y}(n)\right)\right)}{\sqrt{\sum\left(x_{i}(n)-\bar{x}(n)\right)^{2} \cdot \sum\left(y_{i}(n)-\bar{y}(n)\right)^{2}}}
$$

where $x_{\mathrm{i}}$ and $y_{\mathrm{i}}$ are pixel intensities while $\bar{x}$ and $\bar{y}$ are mean intensities measured from outlined plasma membrane segments. The index $n$ refers either to time $t$ or to z-plane number $z$ making the correlation coefficient a function of these parameters ("Results"). By analyzing correlation in 1-dimensional pixel intensity profiles of DHE and DiIC12 along the plasma membrane between the first and 
subsequent images, one obtains a time-dependent correlation coefficient for membrane intensity ("Results").

\section{Quantification of endocytosis and total sterol uptake}

Control and energy depleted $\mathbf{J} 774$ cells were pulse-labeled with DHE/MCD, washed and chased for 30 min either in Medium 1 or Medium 2, the latter containing deoxyglucose and sodium azide. For quantification, two different protocols were used: firstly, three-line scans were made from sum projected stacks at the indicated positions (Fig. 6; "Results"). Mean intensity \pm standard deviation of these line scans were plotted for control and energy-depleted cells. Secondly, quantification of plasma membrane fluorescence of DHE in control and ATP-depleted cells was performed according to the segmentation method developed in our previous work (Wüstner et al. 2005). Briefly, a binary mask was generated for the plasma membrane and for the whole cells from images of DHE labeled cells. These masks were applied to background corrected images of DHE labeled macrophages and the intensities in the defined regions were measured. The ratio of plasma membrane to total cell intensity of DHE gives a relative measure for the fraction of sterol in the plasma membrane being suitable for comparing different experimental conditions like control and ATP-depleted cells.

\section{Fourier and Wavelet analysis of DHE staining patterns}

To determine the periodicities in the 1-dimensional DHE intensity profiles of straightened plasma membrane segments, the periodogram, i.e., the unbiased estimate of the power spectral density of the intensity profiles was calculated using the Time series tool in MatLab (Mathworks, Inc. USA). The periodogram represents the scaled absolute value of the fast Fourier transform of the signal and gives the amplitude of a certain periodicity, the latter expressed in cycles per pixel ("Results"). This analysis provides information about frequencies, i.e., how often a certain intensity pattern occurs in the data, but it lacks spatial information.

The continuous wavelet transform of a linearized signal $f(x)$ (amplitude over distance) is defined as

$$
C_{a b}=W f(a, b)=\frac{1}{\sqrt{|a|}} \int_{-\infty}^{+\infty} f(x) \Psi^{*}\left(\frac{x-b}{a}\right) \mathrm{d} x
$$

where the function $\Psi \in L^{2}$ with $\int_{-\infty}^{+\infty} \Psi(x) \mathrm{d} x=0$ is called a wavelet basis function, or mother wavelet (Blatter 2003). The parameters $a$ and $b$ represent scale and shift in space, respectively, while $\Psi^{*}\left(\frac{x-b}{a}\right)$ means the complex conjugated function to $\Psi$.
Following the protocol of Kobrinsky et al. (2005) a Daubechies wavelet of fourth order (db4) was chosen as mother wavelet (Kobrinsky et al. 2005). These wavelet functions are limits of an iterative process and are bandlimited meaning that they equal zero outside the support. They have compact support and are suitable for local analysis (Blatter 2003; Kobrinsky et al. 2005). The wavelet analysis toolbox implemented in MatLab (Mathworks, Inc., USA) was used to calculate wavelet coefficient matrices $C$ containing the wavelet coefficients $C_{a b}$ according to Eq. 4 . These were used to calculate temporal differences $\left(D_{t}\right)$ in the coefficients according to (Kobrinsky et al. 2005):

$D_{t}=\left(C_{0}-C_{t}\right)^{2}$

Here, $C_{0}$ is the coefficient matrix at time zero (first image in time-lapse sequence), while $t$ is an index representing selected subsequent time points. Thus, the matrix $D_{t}$ contains the squared change in wavelet coefficients between the first image and a chosen subsequent image. Since the wavelet coefficients contain spatial pixel information over the variable $x$ (Eq. 4) as well as frequency information, the expression in Eq. 5 is a measure of local spatiotemporal changes of DHE staining patterns obtained from the time-lapse data (Kobrinsky et al. 2005).

The difference matrices monitoring spatiotemporal changes in membrane staining pattern in the plasma membrane were exported as TIFF images from MatLab (MathWorks, Inc., USA) and plotted from Adobe Photoshop (Adobe Inc., USA).

\section{Results}

Direct visualization and automated tracking of sterol endocytosis in living cells

Long time observation of macrophages was performed by fluorescence time-lapse imaging at $37^{\circ} \mathrm{C}$ right after labeling cells with DHE. This ensured to visualize the earliest events of sterol endocytosis. A representative cell is shown in Fig. 1a-f. Formation of endocytic vesicles was observed at two sites during the first $5 \mathrm{~min}$ of time-lapse imaging (Fig. 1). Sterol containing vesicles occurred initially as invaginated patch followed by budding and scission from the plasma membrane in a time course of about $2.5 \mathrm{~min}$ (Fig. 1b). Endocytic vesicles containing DHE stayed in close proximity to the plasma membrane as revealed by automated object tracking and kymograph analysis (Fig. 1c-f). Once formed, a DHE containing endosome could stay in the cell periphery for several minutes without significant displacement, before it eventually moved back 
towards the plasma membrane (Fig. 1g, and blue track for vesicle 2 in Fig. 1e, f).

From the tracked 2-dimensional vesicle coordinates, the distance moved between individual time steps (Fig. 1h) and the mean square displacement (MSD) were calculated (Fig. 1i). Both the measurements demonstrate that sterol containing endosomes move initially a relative large distance of three to eight pixels corresponding to 0.46 $1.2 \mu \mathrm{m}$ followed by random movement in small displacements. Comparison with image sequence in Fig. 1a-g shows that DHE containing vesicle moves initially away from the plasma membrane towards the cell center. While some vesicles move subsequently back towards the plasma membrane, others become confined or continue to move

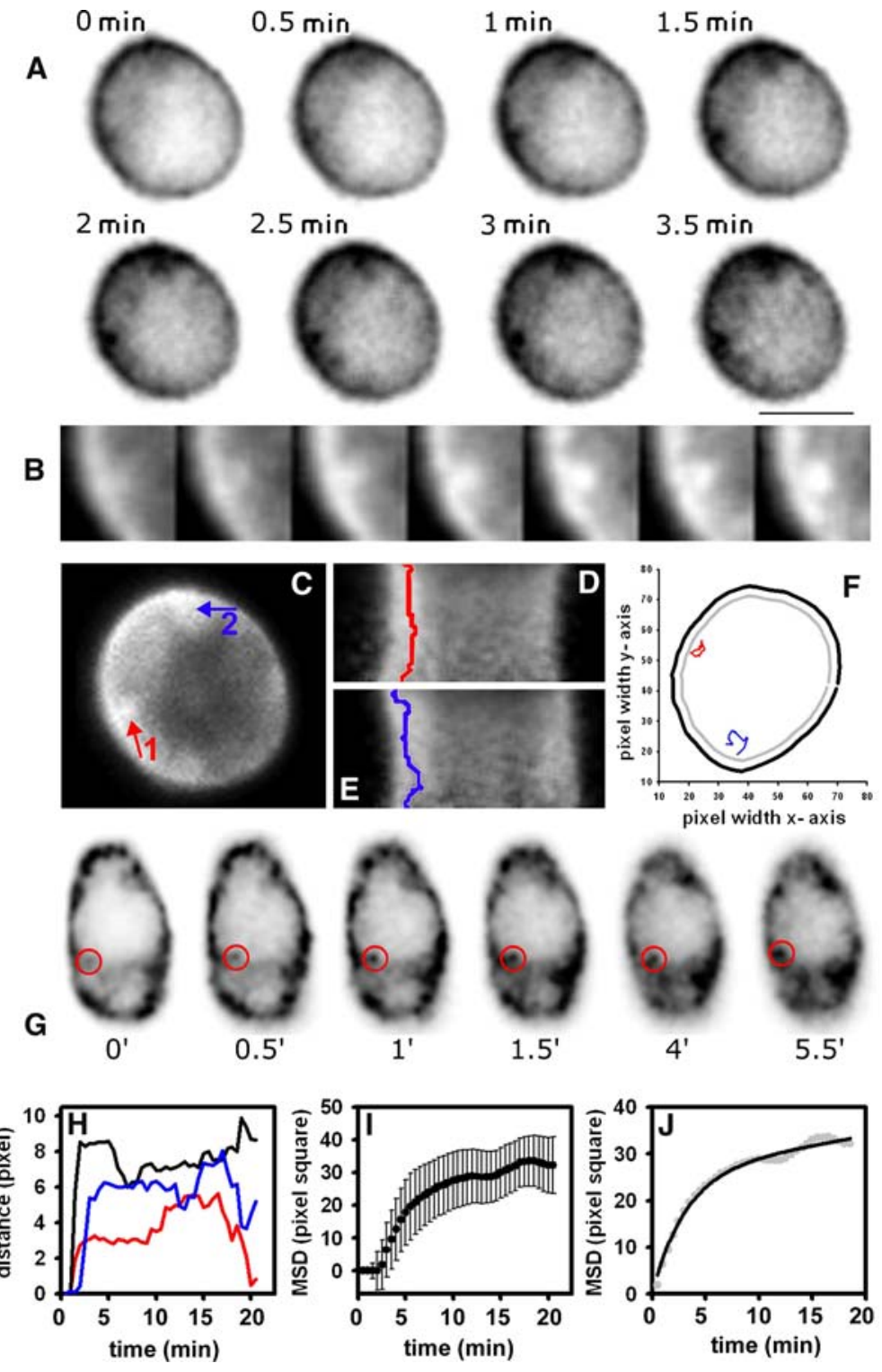

Fig. 1 Time-lapse analysis of sterol endocytosis and tracking of vesicles. J774 cells were pulse-labeled with DHE/MCD for $5 \mathrm{~min}$ at $37^{\circ} \mathrm{C}$, washed and immediately placed on the temperature-controlled stage of a wide field microscope maintained at $35 \pm 1^{\circ} \mathrm{C}$. Images were acquired with $500 \mathrm{~ms}$ acquisition time every $30 \mathrm{~s}$ for a total of 20 min. a Endocytic vesicles containing DHE formed at several places from the plasma membrane. b Direct visualization of vesicle formation in frames acquired every other minute. c Vesicle positions marked for a subsequent tracking analysis (the vesicle shown in b corresponds to vesicle \#1 in red). d, e Kymograph analysis of vesicle trajectories as $x, t$-view for vesicle 1 (red path in d) and $y$, $t$-view for vesicle 2 (blue path in e). f Complete trajectory for both the vesicles tracked over a total time of 20 min suggests confined movement in close proximity to the plasma membrane. $\mathrm{g}$ Another vesicle stayed for up to $5 \mathrm{~min}$ without detectable movement before it returned towards the plasma membrane (red circle). h Distance moved by the three exemplified vesicles as function of time. i Mean square dispplacement (MSD) of all tracked vesicles $(n=5)$ shown as mean \pm SD. $\mathbf{j}$ Non-linear fit of time-dependent MSD to a model of confined diffusion according to Eq. 2. a, g Inverted images. Bar $10 \mu \mathrm{m}$ 
towards the cell center at the end of the observation period (red and blue vs. black line in Fig. 1h). The initial displacement away from the plasma membrane, however, is with $0.5-1 \mu \mathrm{m}$, too large to be accounted for by incomplete vesicle fission after endocytosis. Small subsequent position fluctuations can be well described by a linear function suggesting Brownian motion. The MSD curve was fitted to a model of Brownian diffusion with a 2-dimensional diffusion constant $D$ confined to an area A (Fig. 1j; Eq. 2) (Daumas 2003). Using this model, it was found that endocytic vesicles containing DHE move with $D=1.97 \pm 0.44 \times 10^{-12} \mathrm{~cm}^{2} / \mathrm{s}$ in a confinement area $A=2.3 \pm 0.13 \mu \mathrm{m}^{2}$ in living J774 cells. Alternatively, the initial MSD of vesicles (first five points) was fitted to a linear function according to $\mathrm{MSD}=4 D t$ describing Brownian motion. This gives a diffusion constant of $D=93.6 \pm 0.43 \times 10^{-12} \mathrm{~cm}^{2} / \mathrm{s}$ resembling the initial large movement of endosomes away from the plasma membrane. All tracked vesicles $(n=5)$ were found during the whole observation period of $20 \mathrm{~min}$. There were also vesicles observable for shorter periods like $5 \mathrm{~min}$, but the analysis focussed on long-time tracking. Long trajectories were hard to obtain, presumably due to vesicle movement out of the focus during the experiment. With other fluorescent molecules, like GFP-tagged proteins, this can be prevented by acquiring 4-dimensional stacks; due to the strong bleaching of DHE, however, combined time-lapse and 3-dimensional imaging is not possible for fluorescent sterol. Accordingly, a 2-dimensional model for vesicle diffusion was applied to characterize vesicle motion in the plane of observation (see above). The observed motility pattern of DHE containing endocytic vesicles is in agreement with dynamics of endosomes containing GFP-tagged myosin 6 moving through peripheral actin barriers (Aschenbrenner et al. 2004). In this study, endocytic vesicles moved with $D=1.42 \pm 1.24 \times 10^{-12} \mathrm{~cm}^{2} / \mathrm{s}$ in the cell periphery due to confinement by cortical actin (Aschenbrenner et al. 2004). Secretory granules on growth cones of differentiated PC12 cells showed diffusion constants ranging from $3.9 \times 10^{-11}$ to $2.1 \times 10^{-12} \mathrm{~cm}^{2} / \mathrm{s}$ (Abney et al. 1999). Similarly, motion of secretory granules in the vicinity of the plasma membrane was confined and gave a diffusion constant of $D=2 \times 10^{-12} \mathrm{~cm}^{2} / \mathrm{s}$, very close to that found here for DHE containing endosomes (Steyer and Almers 1999). According to the Einstein hydrodynamic equation $(D=k \mathrm{~T} /(6 \pi \eta r))$ within a viscous fluid $(\eta=0.1$ poise $)$, a vesicle of radius $r=150 \mathrm{~nm}$ would expect to have a diffusion constant of $D=1.4 \times 10^{-9} \mathrm{~cm}^{2} / \mathrm{s}$. Thus, the observed confined diffusion of DHE containing vesicles is still 1000-fold slower than free diffusion in a fluid with ten times the viscosity of water. It is suggested that this is caused by interaction of vesicles with the actin cortex as well as by cytoplasmic crowding (Weiss et al. 2004).
Quantification of time-dependent cell curvature and sterol surface dynamics

The next challenge is to correlate the endocytic activity of cells with dynamics of sterol at the cell surface. We aimed to determine whether sterol endocytosis occurs from certain preformed domains at the plasma membrane or requires lateral sterol enrichment prior to vesicular sterol uptake. Straightening of plasma membrane outlines from a DHE-labeled macrophage extracted from time-lapse sequences allows for determining time-dependent changes in surface staining patterns of DHE (Fig. 2a). Plasma membrane segments resembling the whole cell outlines were generated from images at selected time points and the DHE intensity pattern along the straightened cell perimeter was studied. There is a heterogeneous intensity distribution along each membrane segment (Fig. 2b, c). The positions where formation of endocytic vesicles was observed are indicated, referring to the vesicle numbers of Fig. 1c. Both the vesicles formed in regions of relatively high DHE intensity, but no particular patch of sterol in the size range of the budding vesicles could be detected (compare Fig. 1). Active contour modeling the cell outline of the macrophage shown in Figs. 1a and 2a allows for parallel assessment of the cell shape over the whole observation period (Fig. 2cf). The shown cell is almost round as inferred from the low value of cell elongation. It rounds even slightly up as indicated by the drop in elongation from 0.2 to 0.14 between 7 and 20 min of observation (Fig. 2c). The cell perimeter is almost constant over the whole observation time (Fig. 2d). There are small fluctuations in curvature as measured by active contour tracing of the time-dependent cell shape (Fig. 2f). Membrane activity defined as change of curvature between successive image frames averaged over the whole sequence was almost zero (Fig. $2 \mathrm{~g}$ ). This is in strong contrast to the results obtained in another study with the same contour modeling approach on migrating cells, where curvature changes of more than $40 \%$ strongly oriented towards one side of the cell and more than tenfold increase in cell dispersion were reported in response to stimulation (Dormann et al. 2002). The absence of polarized cell dynamics leads to the conclusion that endocytosis and plasma membrane dynamics of sterol was observed in resting, non-stimulated cells in the current analysis. A nearly constant shape was also found for the other cells used for the time-lapse analysis (not shown). Thus, measured intensity fluctuations of DHE in the plasma membrane (Fig. $2 \mathrm{~b}$ and below) cannot be attributed to overall cell shape changes during the experiment.

Intensity patterns of DHE are stable along the segments over time, as indicated by visual inspection of the preserved staining pattern in Fig. 2b. A quantitative assessment of the DHE distribution can be obtained from a line scan along 

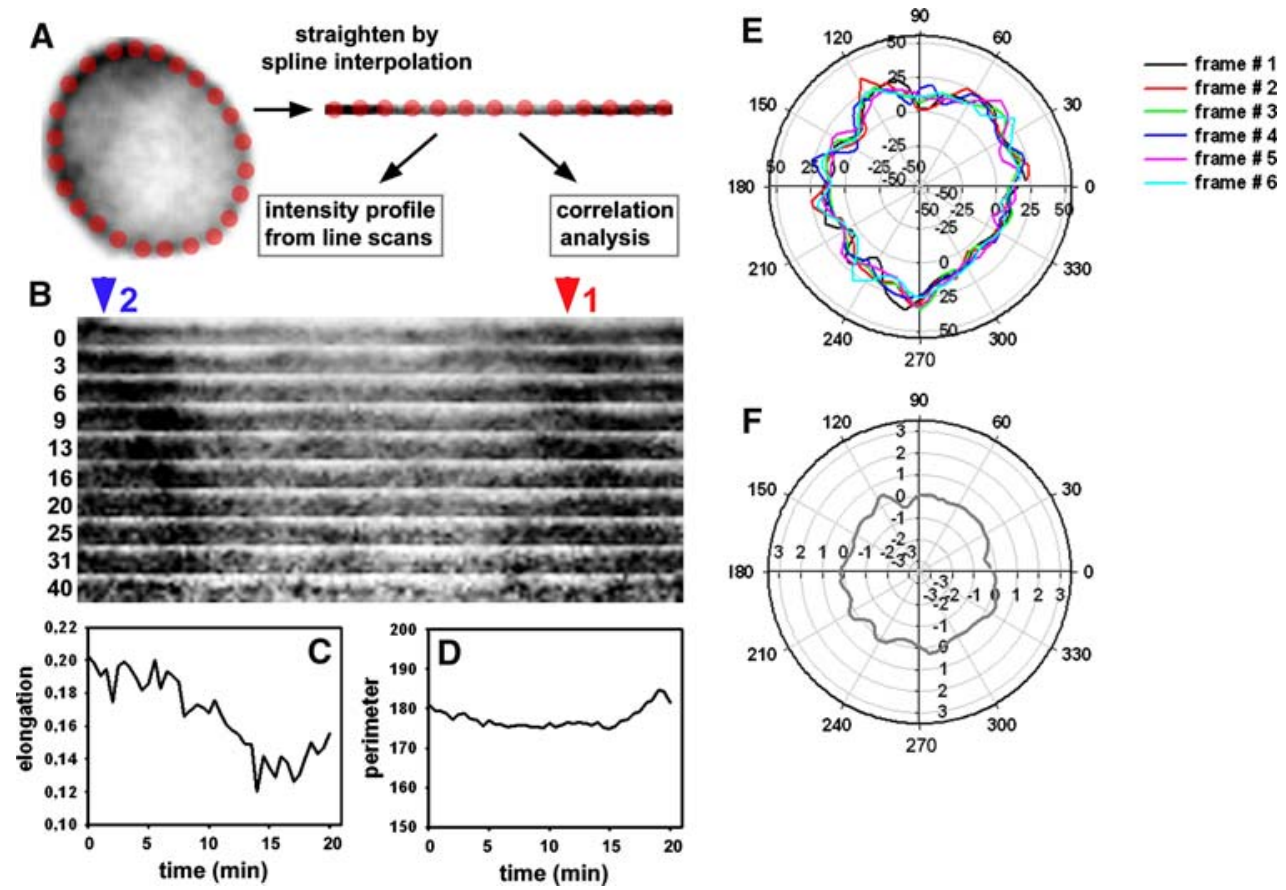

$\nabla 1$
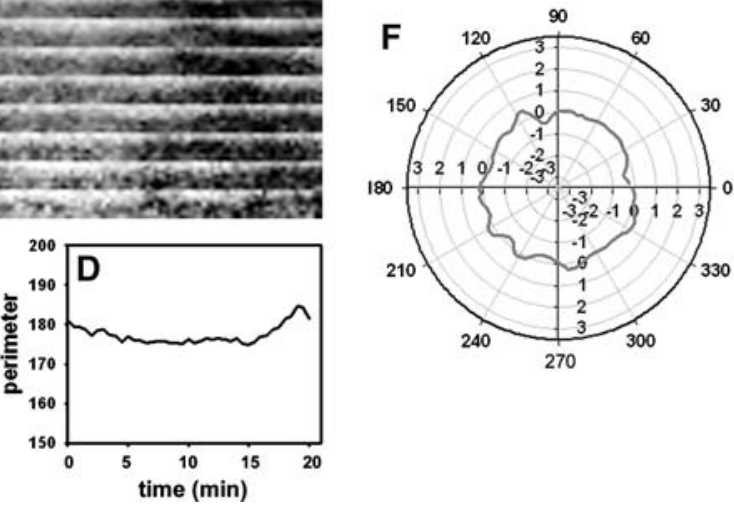

frames being acquired in $30 \mathrm{~s}$ steps. c-f Using an active contour

Fig. 2 Time-dependent plasma membrane staining patterns of DHE and contour tracking of cell surface activity. a From the time-lapse sequence of the J774 cell, shown in Fig. 1a, plasma membrane segments were extracted using a spline interpolation routine implemented in ImageJ. This tool allows for straightening of curved objects and thereby for ease comparison of DHE intensity distribution in the plasma membrane between individual frames. b Aligned straightened plasma membrane (inverted for better visualization) with indicated position were endocytic budding of DHE containing vesicles (compare Fig. 1c, same notation and color). The numbers indicate model, the cell outline was tracked for the cell shown in a. Cell shape parameters, elongation (c) and perimeter (d) were calculated from the cell trajectories and plotted as function of time. This paralleled by measurement of membrane curvature (e) at 100 node positions, shown as polar plot, were the angle codes for node position and the radius for curvature. Local membrane activity can be inferred from plotting the change in curvature per node averaged over the whole trajectory as function of node position (f). a, b Inverted images. See text for further explanations

each straightened segment followed by fitting a sinusoidal function to each data set (Fig. 3a). By calculating the Fourier spectrum as periodogram ("Materials and methods"), a wave like staining pattern of DHE with a period of 147 pixels (0.0068 cycles per pixel) corresponding to $22.6 \mu \mathrm{m}$ was revealed. This period is close to the cell circumference of 182 pixels corresponding to $28 \mu \mathrm{m}$ (compare Figs. 2g, 3b). It remains constant during the whole observation period and resembles the large scale staining pattern of DHE over the cell surface. The periodogram revealed also small amplitudes (about 10\% of the largest period) at higher frequencies with a periodicity of $30-50$ pixels $(0.02-0.03$ cycles per pixel) corresponding to 4.5-7.7 $\mu \mathrm{m}$ (Fig. 3b). This component was observed over the whole image sequence with slightly varying periodicity values in the range of 30-50 pixels. Even smaller fluctuations with amplitude corresponding to $1-2 \%$ of that of the largest period were found on a scale of $\sim 1.0 \mu \mathrm{m}$ (not shown). A correlation analysis of line scan data from extracted plasma membrane segments confirms the analyzed intensity pattern of DHE over time (Fig. 3c): the determined correlation coefficient $R(t)$ declines to a constant value of $R(t)=0.77$ with a half-time of $t_{1 / 2}=2.3 \mathrm{~min}$ as concluded from a fit with a single exponential decay function with residuum to the time dependent correlation coefficient $R(t)$. It is suggested that the constant plateau of $R(t)=0.77$ resembles the large sinusoid staining pattern with a period of more than $20 \mu \mathrm{m}$ being constant over the whole observation period (see above). The other component declining with a half-time of $t_{1 / 2}=2.3 \mathrm{~min}$ from $R(t)=1.0$ to $R(t)=0.77$ is most likely caused by the small scale fluctuations of DHE in the plasma membrane on a scale of $1-8 \mu \mathrm{m}$.

A Fourier analysis of experimental data allows one to identify repeating patterns in the data by harmonic approximation. It does not allow one to determine where these periodicities occur in the intensity data profile, or when they are found in case of time series. In other words, Fourier analysis provides frequency but lacks spatial information, or, in case of time series analysis, temporal information. A simple and easy analogy is found in music where a score contains information about the notes to be played (frequency information) as well as about time (when notes to be played). Fourier transformation of a signal reveals the frequencies, while temporal (time series) 

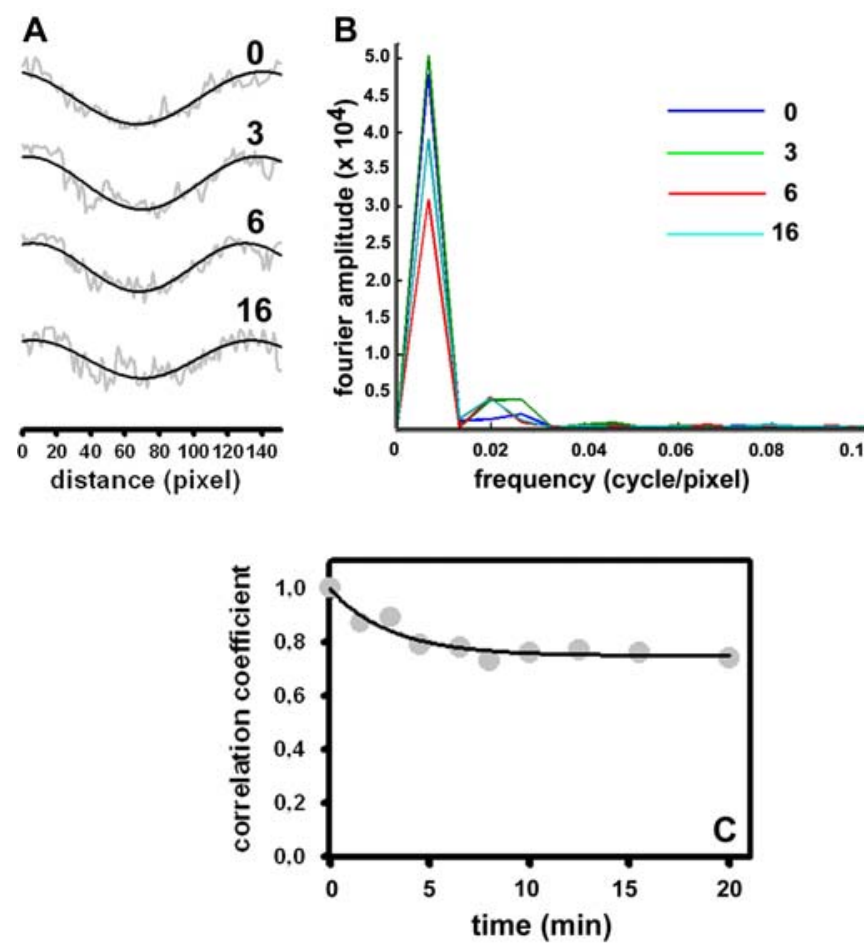

Fig. 3 Statistical analysis of time-dependent DHE membrane staining patterns from extracted and straightened plasma membrane segments, as shown in Fig. 2b 1-dimensional line scans, were calculated and analyzed. a Line scan along the straightened segments is shown in grey, while the black wave curve indicates a fit of the fluorescence data $y(x)$ as function of pixel position $x$ to a sinus function according to $y(x)=y_{0}+a \sin (2 \pi x / b+c)$. b Periodogram of the line scan data reveals periods at 0.0068 and $0.02-0.03$ cycles per pixel corresponding to the 147 pixels $(22.6 \mu \mathrm{m})$ and $30-50$ pixels $(4.6-7.7 \mu \mathrm{m})$ for a full cycle, respectively. The number indicates frame (segment) number $(0-16$, corresponding to $0-8 \mathrm{~min})$. c Correlation coefficient $R$ as function of time calculated for the first segment (termed ' 0 ' in a indicating the first frame of the time-lapse sequence) and in all the following frames. Grey symbols data, black line fit to a single exponential decay function $f(t)=A \exp (-k t)+B$. From this fit, a correlation time indicating the half time of correlation

or spatial information (line scan data) is lost. By analogy with music this would mean that information about notes is maintained after transformation while information about when the notes should be played is lost. In contrast, a wavelet analysis allows for localizing spectral components assigned to a certain wavelet as function of pixel position $x$ or time increment $t$ in case of spatially and temporally varying signals, respectively (see "Materials and methods" and (Blatter 2003; Kobrinsky et al. 2005; Mager and Abernethy 2007) for a detailed discussion). Again, in musical terms, the whole composition can be recovered from the wavelet transformation because it contains all information found in the original score. The wavelet
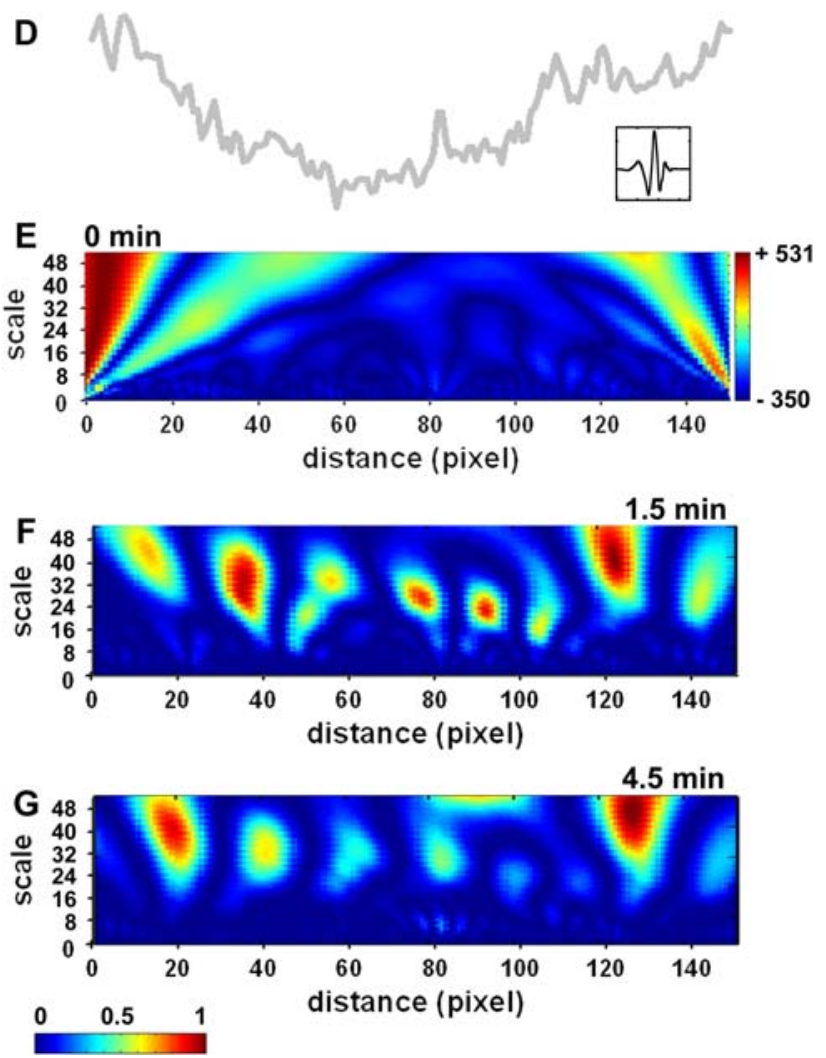

decay can be calculated according to $\tau=\ln 2 / k$ giving $\tau=2.1 \mathrm{~min}$. d-g Wavelet analysis of data. $\mathbf{d}$ Zoomed 0-min-line scan compared to the used Daubechies wavelet of fourth order (db4) (squared box). e Matrix of wavelet coefficients color coded according their values as calculated by a continuous wavelet transform of the 0-min-line scan data. f, g Scaled difference matrices are calculated by subtracting each matrix coefficient according to Eq. 5 in "Materials and methods". Only matrices after $1.5 \mathrm{~min}$ (frame 3 ) and $4.5 \mathrm{~min}$ (frame 9) are shown; but see Supplemental Data for further results. Areas with high coefficient differences according to Eq. 5 in $\mathbf{f}$ and $\mathbf{g}$ highlight membrane regions with transient heterogeneous DHE staining compared to the first frame (0-min line scan). The coefficient difference matrices were normalized to the maximal value giving a color coding for the highest difference that is equal to 1 (red) and for the low difference approaching 0 (blue)

approach has been previously applied to analysis of plasma membrane signaling domains using fluorescence resonance energy transfer microscopy (Kobrinsky et al. 2005). DHE intensity profiles shown in Fig. 3a, d were subjected to a continuous wavelet transform (Fig. 3e). Temporal differences between wavelet coefficient matrices were calculated to identify local heterogeneities of DHE staining patterns (yellow to red regions in Fig. 3f, g). J774 cells showed episodes of transient heterogeneity in plasma membrane staining of DHE. Interestingly, this transient heterogeneity was confined to certain membrane regions like around pixel position 20,40 and 60 , respectively. Due to the spatiotemporal resolution of the wavelet analysis it can be 
concluded that these heterogenous regions did not exceed 15 pixels in diameter (corresponding to $2-3 \mu \mathrm{m}$ ), while they lasted between 3 and 15 min. Thus, wavelet analysis reveals new information not obtainable from correlation or Fourier analysis, namely that some small scale intensity patterns in the size range of $2-3 \mu \mathrm{m}$ are confined to discrete regions of the plasma membrane and exist over up to 15 min (see Supplemental Data).

Together, these results demonstrate that the spatiotemporal plasma membrane distribution of DHE can be described by a constant pattern in the range of $10-20 \mu \mathrm{m}$ being stable over the whole observation period of $20 \mathrm{~min}$. Additionally, the analysis shows that there are small fluctuations in the DHE signal with typical sizes of 2-3 $\mu \mathrm{m}$ and varying duration. This spatiotemporal behaviour is typical for cell surface activity caused by cell protrusions like filopodia, coated pits and microvilli. It is not in line with rapidly diffusing lateral sterol domains in the bilayer plane. To further sustain this conclusion, a fluorescent lipid probe, DiIC12, was used. This probe was shown to partition preferentially into fluid phase domains in model membranes (Spink et al. 1990). In fact, our recent work demonstrates that in giant unilamellar vesicles (GUV) consisting of dioleoylphosphatidylcholine (DOPC), dipalmitoylphosphatidylcholine (DPPC) and DHE in a ratio of 1:1:1 labeled additionally with $0.5 \mathrm{~mol} \%$ DiIC12, the fluorescent sterol is able to induce liquid-liquid immiscibility and partitions almost exclusively into the ordered phase, while DiIC12 partitions into the liquid-disordered phase completely segregating from DHE (Garvik et al. 2008). In the plasma membrane of living cells, DiIC12 has a homogeneous distribution at the level of the light microscope (Wüstner 2007b). Thus, DiIC12 should stain all regions of the macrophage cell membrane, and dynamics of this probe should monitor surface activity but not putative liquid ordered domains in the cell membrane. Time-lapse imaging of DiIC12 labeled J774 cells demonstrates the dynamics of filopodia and membrane ruffles at the cell surface (Fig. 4a, b). Again, a resting macrophage was chosen for the analysis as indicated by active contour tracing of cell outlines (Fig. 4c, d). The investigated cell rounds up slightly as indicated by the slight decrease in cell elongation (Fig. 4d). The staining pattern obtained from a line scan of the plasma membrane reveals a very stable staining pattern of DiIC12 with a high time-dependent correlation coefficient. The determined correlation coefficient $R(t)$ declines to a stable plateau with a half-time of $t_{1 / 2}=1.6 \mathrm{~min}$ as concluded from a fit with a single exponential decay function with residuum (see above for DHE). Note that the image-to-image correlation in DHE and DiIC12 staining patterns, respectively, is very high (above $R=0.85$ ) and constant over the whole sequence (shown for DiIC12 in Fig. 4d). Moreover, a wavelet analysis of DiIC12's staining pattern showed the same characteristics as that of DHE (see Supplemental Data). The similarity of DHE's dynamic membrane staining pattern with that of the lipid probe DiIC12 strongly suggest that sterol dynamics at the cell surface resemble dynamic changes of the surface topology caused by ruffles, microvilli and other protrusion types.
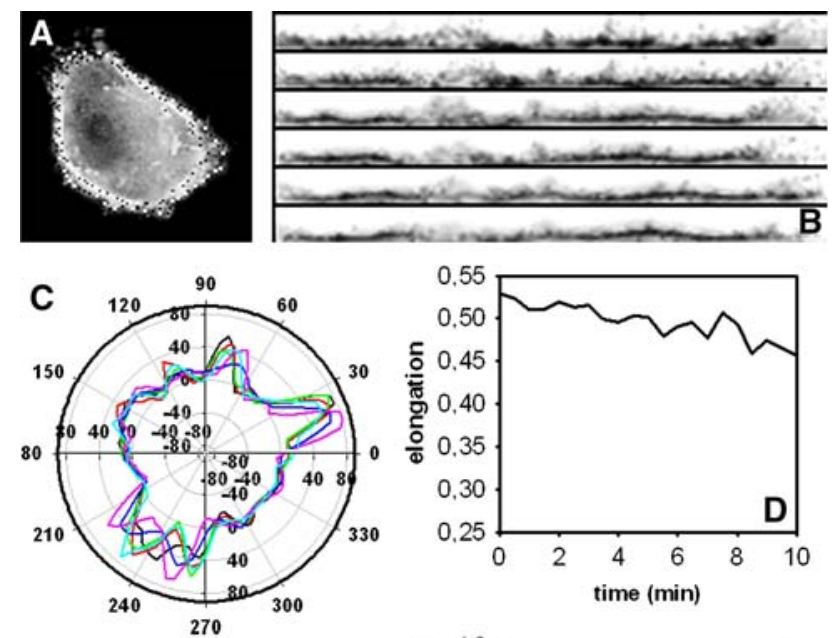

E
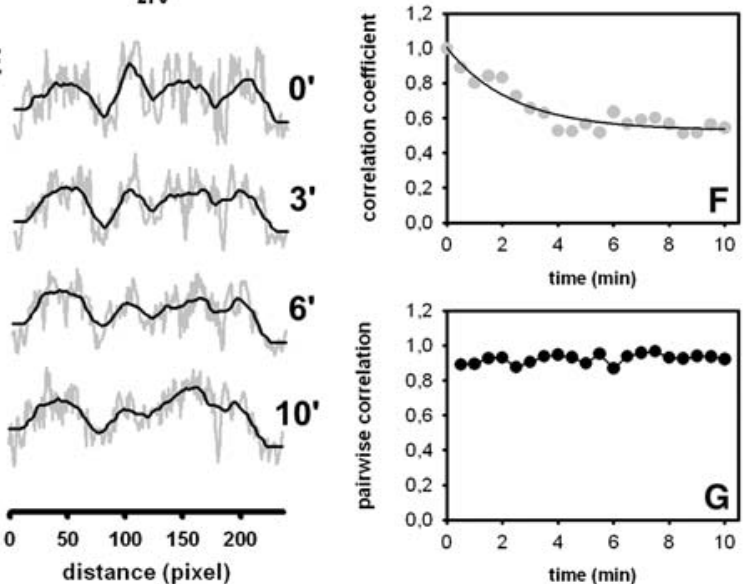

Fig. 4 Time-dependent plasma membrane staining patterns of a fluorescent membrane marker. J774 cells were labeled with DiIC12 for $1 \mathrm{~min}$ at $37^{\circ} \mathrm{C}$, washed and immediately placed on the temperature-controlled stage of a wide field microscope maintained at $35 \pm 1{ }^{\circ} \mathrm{C}$. Images were acquired every $30 \mathrm{~s}$ for a total time of 10 min. b Plasma membrane segments were extracted using a spline interpolation routine implemented in ImageJ (inverted image is shown). c An active contour model applied to the cell outline was used to measure cell curvature for selected frames as well as for determining cell elongation (d). e Line scan along the cell membrane is shown in grey, while the black line shows the data smoothed with a running average filter implemented in SigmaPlot 9.0 (SPSS Inc., Chicago, IL, USA). f Correlation coefficient $R$ as function of time calculated between the first and all subsequent line scan data obtained from the time lapse sequence. Grey symbols data, and black line fit to a single exponential decay function $f(t)=A \exp (-k t)+B$. From this fit, a correlation time indicating the half time of correlation decay can be calculated according to $\tau=\ln 2 / k$ giving $\tau=1.6 \mathrm{~min}$. $\mathbf{g}$ Pair wise correlation between line scans derived from successive time lapse frames 


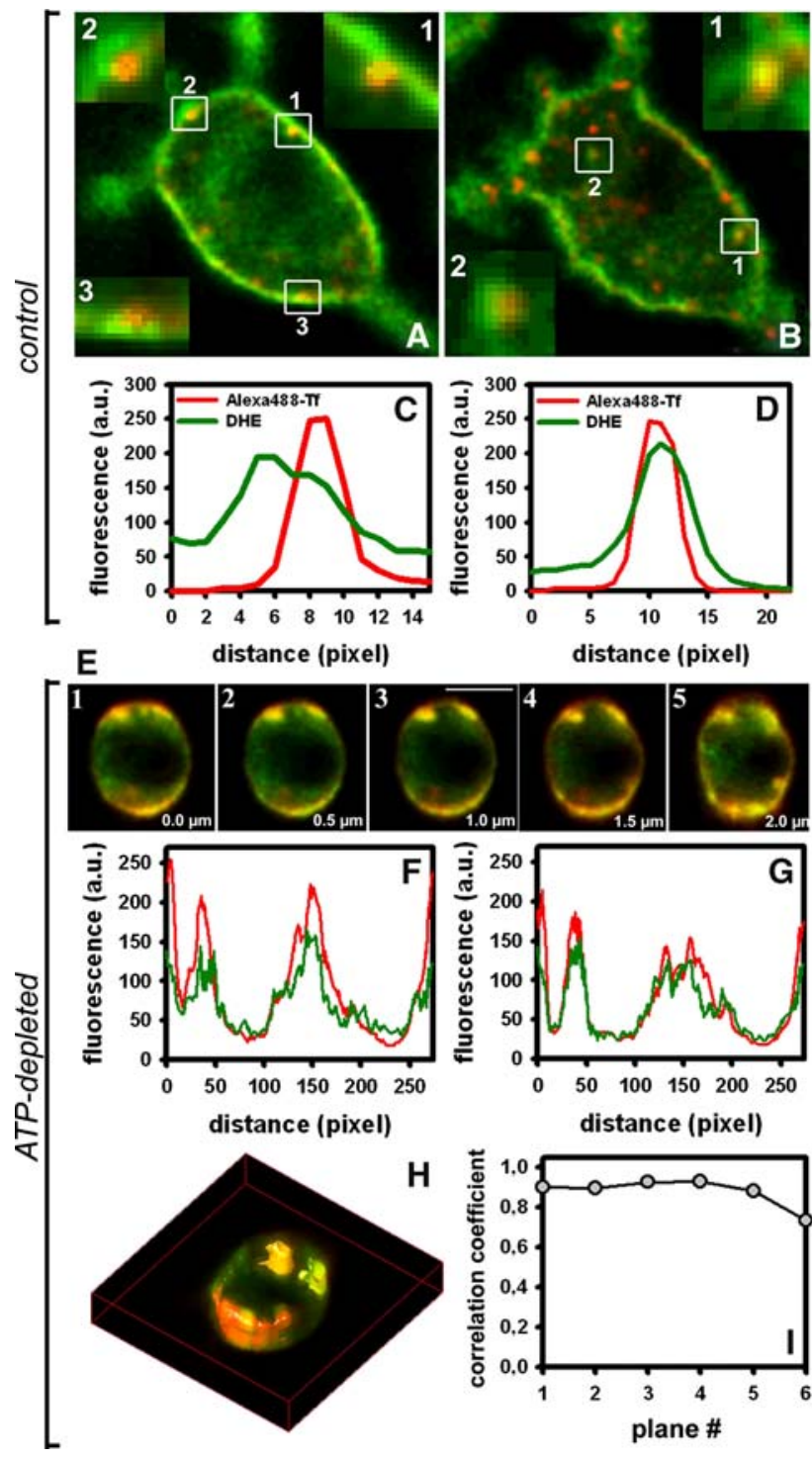

DHE co-localizes with transferrin in endocytic vesicles and at the plasma membrane

Time-lapse imaging allows for visualization of sterol endocytosis together with analysis of dynamic changes in surface distribution of fluorescent sterol. It does not provide information regarding the identity of forming endocytic vesicles containing DHE. This information can be provided by pulse-chase experiments of DHE together with known endocytic markers. We showed recently, that DHE co-localizes with Alexa546-Tf in sorting endosomes and in the ERC in J774 cells after 5 and 30 min chase, respectively (Wüstner et al. 2005). This result does not necessarily mean that DHE is also internalized together with $\mathrm{Tf}$ by clathrin-mediated endocytosis. It is possible that DHE containing endosomes fuse with Tf containing sorting endosomes at later time points. As shown in Fig. 5a-d,
4 Fig. 5 Co-localization of DHE with Alexa546-Tf in control and ATP-depleted cells. a-d J774 cells were incubated for $5 \mathrm{~min}$ at $37^{\circ} \mathrm{C}$ with DHE/M $\beta$ CD containing $10 \mu \mathrm{g} / \mathrm{ml}$ Alexa 546-Tf, washed with ice-cold Medium 1, fixed with $2 \%$ paraformaldehyde (PFA) for $10 \mathrm{~min}$ on ice, washed extensively and imaged on a wide field microscope. Image stacks of double-labeled cells were acquired with $0.5 \mu \mathrm{m}$ distance followed by background correction and 3-dimensional deconvolution using Hyugens software (SVI, Hilversum, The Netherlands). DHE (green) co-localized occasionally with Alexa546$\mathrm{Tf}$ ( red) in vesicles adjacent to the plasma membrane (indicated by boxes, see inset in $\mathbf{a}, \mathbf{b})$. c, $\mathbf{d}$ Line scan across vesicle containing DHE and Alexa546-Tf from box 2 in $\mathbf{a}(\mathbf{c})$ and box 1 in $\mathbf{b}(\mathbf{d})$ reveals exact overlay of both probes in the point-like objects. $\mathbf{e}-\mathbf{i}$ Cells were labeled like described for control cells in a-d, except that they were first incubated for $30 \mathrm{~min}$ at $37^{\circ} \mathrm{C}$ in Medium 2 containing sodium azide and 2-deoxyglucose, followed by labeling and fixation (for alternative ATP-depletion protocol, see "Materials and methods"). Co-localization of DHE with Alexa546-Tf was determined in ATP-depleted J774 cells as described for control cells, except that all incubations were performed in Medium 2. e Single deconvolved planes of a stack of double-labeled cells with DHE in green and Alexa546-Tf in red. f, $\mathbf{g}$ Line scans along the plasma membrane for two selected planes from the stack show exact overlay of both staining patterns. Scan for plane 1 (f) and plane 4 (g). h Three-dimensional reconstruction of colocalization volume from the whole stack. i Bivariate correlation coefficient between the 1D staining pattern of DHE and Alexa546-Tf as a function of $z$-distance along the stack

DHE co-localizes with Alexa546-Tf at earliest time points of endocytosis. Representative corresponding planes of spatially registered, 3-dimensional deconvolved stacks of DHE and Alexa546 double-labeled cells reveal clearly that some, but not all Alexa546-Tf containing endosomes contain also DHE. These early endocytic vesicles were found in close proximity to the plasma membrane and formed over the whole cell thickness as exemplified on two planes acquired $2 \mu \mathrm{m}$ apart along the optical axis (Fig. 5a, b). Fluorescent sterol does not only co-localize with Alexa546-Tf in early endosomes but also at the cell surface: DHE and Alexa546-Tf were found in patches of the plasma membrane as revealed in ATP-depleted cells, where clathrin-dependent endocytosis is blocked (Fig. 5e). Double-labeled patches appeared as large invaginations of the macrophage cell surface across the whole 3-dimensional volume (Fig. 5e-h). The lateral distribution of fluorescent sterol showed strong correlation with that of labeled $\mathrm{Tf}$ as indicated by a correlation coefficient $R(z)>0.85$ over a $z$-distance of $2.5 \mu \mathrm{m}$ (Fig. 5i). These results demonstrate that a portion of DHE is collected in clathrin-coated pits together with fluorescent $\mathrm{Tf}$ followed by common internalization of both the probes.

Endocytosis contributes by more than $60 \%$ to total sterol uptake in macrophages

Endocytosis is an energy-requiring process, while nonvesicular sterol uptake occurs also in the absence of 


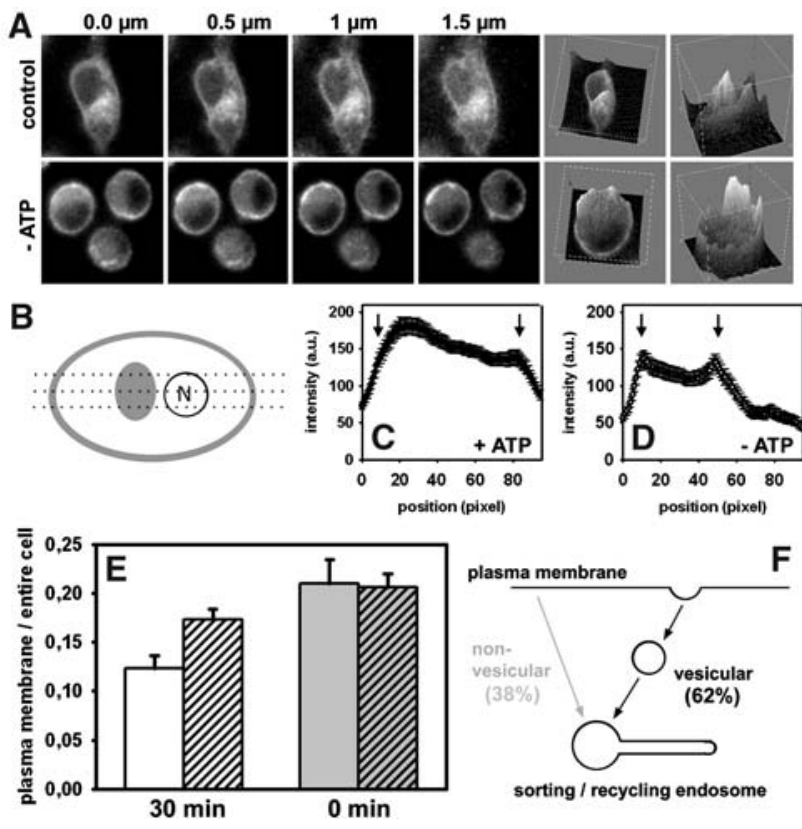

Fig. 6 Quantification of vesicular and non-vesicular sterol uptake in $\mathrm{J} 774$ cells. After pulse-labeling with DHE/MCD for $5 \mathrm{~min}$ at $37^{\circ} \mathrm{C}$, normal and ATP-depleted cells were washed and imaged in $z$-stacking modus on a wide field microscope. Control cells showed a prominent enrichment of DHE in the perinuclear region, while energy-poisoned cells had highest DHE intensity in the plasma membrane accompanied by strong diffuse intracellular staining. a Individual planes (left eight panels) and two views of a 3-dimensional reconstruction (right four planes) of control (upper panels) and ATP-depleted cells (lower panels) acquired in $z$-stacking modus. b Scheme for positioning of line scans relative to the cell. c, d Mean and standard deviation for three-line scans measured according to scheme in b for control (c, “+ATP”) and energy-depleted cells (d, “-ATP”). e Quantification of plasma membrane associated DHE normalized to total cell DHE for control (open bars) and ATP-depleted cells (crossed bars) right after pulse-labeling (grey bars, "0 min") or after $30 \mathrm{~min}$ chase at $37^{\circ} \mathrm{C}$ (white bars, "30 min"). f Relative contribution of vesicular endocytic uptake $(62 \%)$ versus non-vesicular uptake $(38 \%)$ of sterol calculated from this data. Data in e represent mean \pm standard deviation of at least 15 independent measurements per time point. See text for further explanations

cellular ATP (Mukherjee 1997; Wüstner et al. 2005). By comparing plasma membrane versus intracellular fluorescence of DHE in control and ATP-depleted cells, it will be possible to quantify the contribution of endocytosis to total cellular sterol uptake from the plasma membrane. As shown in Fig. 6, ATP-depletion results in an altered intracellular distribution of DHE in macrophages with strongly reduced accumulation of DHE in the perinuclear area compared to control cells. Vice versa, plasma membrane fluorescence of DHE is significantly higher in energy-poisoned cells as easily inferred from a 3-dimensional reconstruction of the stacks (Fig. 6a, right panels). To assess quantitatively the effect of ATP-depletion on sterol uptake, three-line scans were made from sum projected stacks at the indicated positions (Fig. 6b). Mean intensity \pm standard deviation of these line scans plotted for control and energy-depleted cells support that ATP is required for accumulation of DHE in the perinuclear area in J774 cells (Fig. 6c, d; arrows indicate position of the plasma membrane). Quantification of plasma membrane fluorescence of DHE in control and ATP-depleted cells by the segmentation method developed in our previous work confirms this conclusion (Fig. 6e) (Wüstner et al. 2005). From this, the fraction of DHE in the plasma membrane compared to total cell-associated DHE can be estimated for different conditions ( \pm ATP) after $30 \mathrm{~min}$ chase and compared to the initial membrane fluorescence after $0 \mathrm{~min}$ chase. This fraction is 58.6 and $84.3 \%$ of initial in control and energy-depleted cells, respectively. Thus, 41.37 and $15.7 \%$ of plasma membrane sterol become internalized in control and ATP-poisoned cells, respectively; indicating that endocytosis contributes by $62 \%$ to DHE uptake from the cell surface in macrophages. The results presented in Fig. 5 suggest that at least a portion of this vesicular sterol uptake occurs by clathrin-dependent endocytosis together with Tf, a marker for this pathway.

Fluorescent sterols do not accumulate in cell surface caveolae but caveolar endocytosis contributes to sterol uptake from the plasma membrane

Caveolae are flask-shaped surface invaginations of 50$100 \mathrm{~nm}$ diameters, being abundant in certain cell types like smooth muscle cells and adipocytes (Ikonen 2000). These surface regions are proposed to trigger cell surface signaling, and internalization of caveolae is a pathway for cell invasion of certain viruses (Parton 2003). Caveolae contain the membrane protein caveolin, and expression of caveolin triggers the formation of caveloae (Ikonen 2000). Caveolin binds cholesterol and caveolae have been proposed to be specifically enriched in cholesterol and certain sphingolipids eventually playing a role in cholesterol uptake from the cell surface (Simons and Ikonen 1997). If caveolae contain indeed higher amounts of sterols than the rest of the plasma membrane, we should be able to see an enrichment of fluorescent sterols in caveloae. To test this hypothesis, we looked first at adipocytes which form many caveolae at their cell surface, and where caveloae cluster to large microscopically visible macrodomains called caves (Parton et al. 2002). 3T3 L1-adipocytes were triple-labeled with DHE, the fluid phase preferring lipid probe DiIC12 and the ganglioside binding toxin Alexa488-CtxB (Fig. 7a). All three probes exactly coincided in caveolar structures. To selectively label adipocyte caveloae, we transiently expressed a GFP-tagged caveolin 1 (GFP-Cav1) in 3T3 L1-adipocytes ("Materials and methods"). In accordance with previous results, we found fluorescent caveolin 1 in plane regions of the plasma membrane as well 

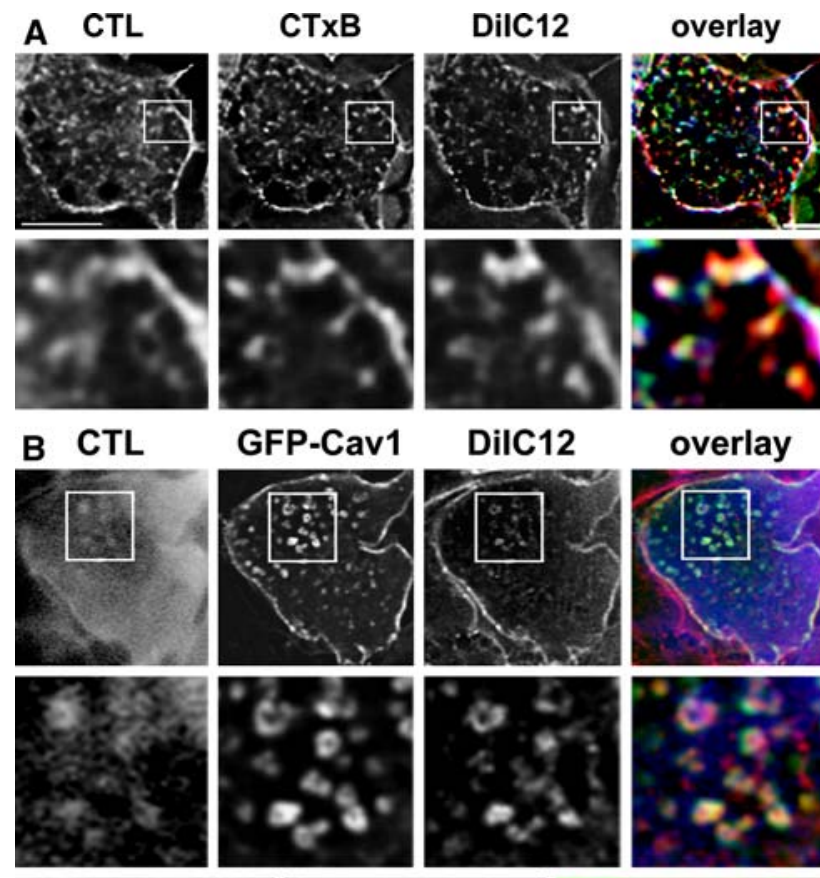

overlay
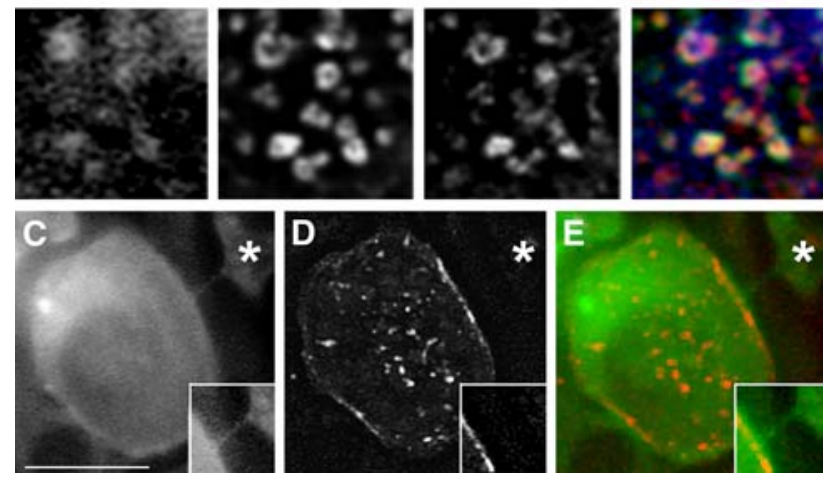

Fig. 7 Fluorescent sterol is not enriched in caveolae and surface caves in adipocytes. a 3T3-L1 adipocytes were labeled with DHE/ MCD for $2 \mathrm{~min}$ at $37^{\circ} \mathrm{C}$, washed and pulse labeled with DiIC12, placed on ice and labeled with $50 \mu \mathrm{g} / \mathrm{ml}$ Alexa488-CTxB. Cells were washed once with ice-cold and twice with warmed Medium 1 and imaged on a wide field microscope. Images were 2-dimensional deconvolved using a deblurring algorithm implemented in the Huygens software (Scientific Volume Imaging, Hilversum, The Netherlands). Lower panel is a zoom of the box shown in the upper panels. Color overlay shows DHE in blue, Alexa488-CTxB in green and DiIC12 in red. b-d Adipocytes expressing transiently GFP-Cav1 were labeled with CTL/MCD and DiIC12, washed and imaged as described above. The focus was set to the bottom of cells to visualize ring-like adipocyte surface caves (Parton et al. 2002). These caves were strongly labeled by GFP-Cav1 as well as by DiIC12 and some CTL, but sterol was not enriched in these structures (see inset in lower panel of b). c-d Preadipocytes expressing GFP-Cav1 showed patches of GFP fluorescence at the cell bottom and rim (d). In contrast, CTL was homogenously distributed over the whole cell surface (c), and labeled also a nanotube connecting two cells which lacked GFP-Cav1 (star in c-e, and inset). Color overlay (e) shows fluorescent sterols in green and GFP-Cav1 in red. Bar $10 \mu \mathrm{m}$

as in large ring-like surface caves at the bottom of mature adipocytes (Fig. 7b) (Parton et al. 2002). These adipocytespecific structures have been shown to contain beside caveolin also GLUT4, cholera toxin bound to ganglioside, clathrin, transferrin receptor as well as syntaxin (Parton et al. 2002). In GFP-Cav1 expressing 3T3 L1-adiopocytes
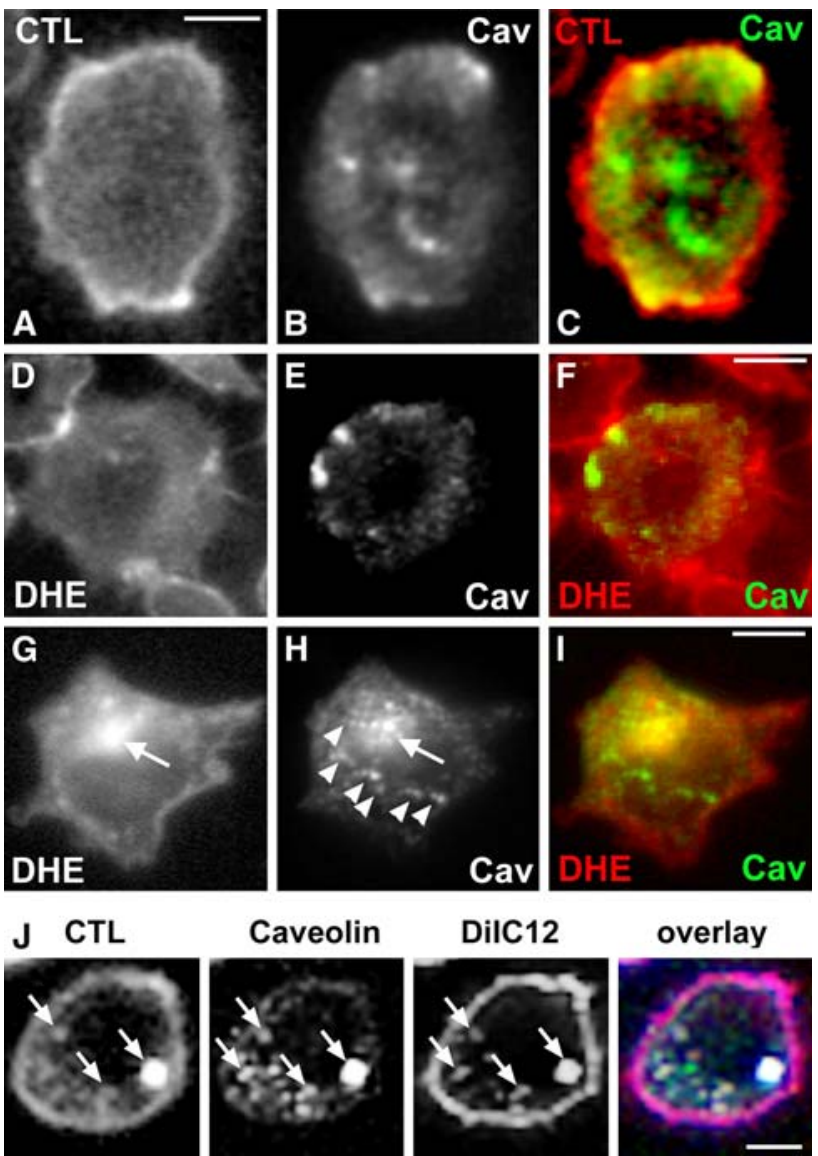

overlay

Fig. 8 Plasma membrane sterol is homogeneously distributed and partially internalized by caveolar endocytosis in $\mathrm{CHO}$ cells expressing GFP-Cav1. CHO cells expressing GFP-Cav1 were either labeled with CTL/MCD $(\mathbf{a}-\mathbf{c})$ or with DHE/MCD $(\mathbf{d}-\mathbf{i})$ for 2 min at $37^{\circ} \mathrm{C}$, washed and imaged on a wide field microscope with the focal position centered at the bottom of the cells close to the glass interface (a-f) or chased for another $30 \mathrm{~min}$ before imaging an equatorial plane of the cell $(\mathbf{g}-\mathbf{i})$. CTL (a), DHE (d, g) and GFP-Cav1 $(\mathbf{b}, \mathbf{e}, \mathbf{h})$. Color overlay (c, f, i) shows fluorescent sterols in red and GFP-Cav1 in green. $\mathbf{j}$, GFP-Cav1 expressing CHO cells were labeled with CTL/MCD and DiIC12 as described above, washed and chased for $5 \mathrm{~min}$ at $37^{\circ} \mathrm{C}$. Some vesicles containing CTL, caveolin as well as DiIC12 were found in the peripheral cytoplasm indicating that they were internalized together by endocytosis. Bar $5 \mu \mathrm{m}$

co-stained with the fluid phase marker DiIC12 and with CTL we observed no enrichment of the fluorescent sterol in these caveolin-rich surface caves compared to DiIC12. Both the lipid probes stained these surface structures, but there was no particular accumulation of CTL in regions being strongly labeled by GFP-Cav1 (insets of Fig. 7b). Caveolin containing surface caves are a specialization of mature adipocytes but are not present in preadipocytes (Parton et al. 2002). In the latter cells we found GFP-Cav1 in spots or patches distributed over the cell surface and at the cell edge, but these regions were again not particularly enriched in CTL. The sterol but not GFP-Cav1 showed homogeneous staining of the plasma membrane in 
preadipocytes (Fig. 7c-e). Together, these results demonstrate that surface caves and caveolae of adipocytes do not have higher sterol content than the rest of the plasma membrane.

Next, we asked whether the absence of sterol enrichment in caveolae is specific for adipocytes. GFP-Cav1 was expressed in $\mathrm{CHO}$ cells and the distribution of this protein was compared to that of the fluorescent sterols CTL (Fig. 8a-c) or DHE in the plasma membrane (Fig. 8d-f). GFP-Cav1 was found in patches at the cell surface probably resembling single or clustered caveolae. Neither CTL nor DHE, however, did co-patch with GFP-Cav1; both sterols showed a homogeneous distribution over the cell surface of $\mathrm{CHO}$ cells as found for adipocytes. It has to be emphasized that even if caveolae are smaller than the resolution limit of the wide field fluorescence microscope, this will not prevent us from determining sterol intensity in these surface specializations. The resolution limit of the fluorescence microscope given by the Rayleigh criterion defines the minimal distance between two point-like objects which can be resolved by diffraction limited optics: $\mathrm{d}(\mu \mathrm{m})=0.61 \lambda /(n \sin \alpha)$ in the focal plane with $\lambda, n$, and $\alpha$ denoting the wavelength, refractive index and the aperture angle of the lens, respectively (Inoue 1997). With our UV sensitive imaging system, with a numerical aperture of the objective $n \cdot \sin \alpha=\mathrm{NA}, \mathrm{NA}=1.4$, and $\lambda=380 \mathrm{~nm}$ we are theoretically able to resolve "fluorescent sterol spots" in the membrane being as close as $d=165 \mathrm{~nm}$, while for GFP-Cav1 $(\lambda=540 \mathrm{~nm})$ we would have a minimal distance of $d=235 \mathrm{~nm}$. Due to the low contrast in images of DHE or CTL, however, this theoretical limit cannot be achieved in praxis. This, however, is also not a requirement. Even if we cannot resolve individual caveolae, we are able to judge whether a membrane region or patch of the typical size of approximately $250-500 \mathrm{~nm}$ labeled by GFP-Cav1, and by this criterion defined as caveolar region, has a relative enrichment of fluorescent sterol over the rest of the membrane. The data clearly demonstrate that fluorescent sterols which resemble cholesterol very closely do not become enriched in cell surface caveolae in living cells.

To determine whether caveolae mediate endocytosis of sterols from the cell surface, endocytic vesicles have to be specifically highlighted. We double-stained GFP-Cav1 expressing CHO cells with DiIC12 and CTL by a short labeling pulse, washed the cells and chased them for $5 \mathrm{~min}$ at $37^{\circ} \mathrm{C}$ to allow for co-internalization of both the probes (Fig. 8j). There were a few endocytic vesicles containing CTL, DiIC12 as well as GFP-Cav1 suggesting that some sterol can be internalized via caveolar endocytosis. In fact, caveolae have been shown to be immobile at the plasma membrane and endocytosis of caveolin was found to be a rare event under basal conditions (Thomsen 2002). In a separate experiment, GFP-Cav1 expressing cells pulse- labeled with CTL were chased for $30 \mathrm{~min}$ at $37^{\circ} \mathrm{C}$ (Fig. 8g-i). We found some co-localization of CTL and GFP-Cav1 in the perinculear ERC pool (arrows, Fig. 8g-i). A large portion of intracellular sterol, however, did not colocalize with fluorescent caveolin (arrowheads, Fig. 8g-i).

\section{Discussion}

This study describes the first visualization of the dynamic process of sterol endocytosis and investigates the associated changes in plasma membrane sterol distribution in living mammalian cells. By time-lapse imaging it was possible to directly observe formation and budding of endocytic vesicles containing the intrinsically fluorescent close cholesterol analog DHE from the cell surface. To this end, new image analysis tools including noise robust tracking of vesicle paths were applied. Vesicle budding could be correlated with plasma membrane dynamics of DHE while curvature fluctuations of the cell were determined in parallel. DHE containing vesicles co-localized with fluorescent $\mathrm{Tf}$ at the earliest time points of internalization. In ATP-depleted cells, uptake of Tf was completely blocked and internalization of DHE strongly reduced (by $62 \%$ compared to control cells, see Fig. 6) indicating that endocytosis is the dominant pathway of sterol uptake in macrophages. We found a quantitatively similar effect of ATP depletion on internalization of DHE in hepatoma cells, suggesting that the extent of sterol endocytosis differs only slightly between various mammalian cell types (Hartwig Petersen et al. 2008). Note that spontaneous transfer of DHE between model membranes in the fluid phase is almost five times faster than that of cholesterol [i.e., half-time of $14 \mathrm{~min}$ for DHE compared to $65 \mathrm{~min}$ for cholesterol in vesicles made of POPC at $37^{\circ} \mathrm{C}$ (Bar 1989)]. Transfer characteristics are the same for DHE and cholesterol (i.e., single exponential exchange kinetics and existence of a nontransferable pool of comparable size). From these results, it can be expected that cholesterol has a significantly lower rate of non-vesicular and potentially a higher fraction of vesicular uptake from the plasma membrane than DHE. Together, these results support a model where endocytosis is the dominant internalization pathway for cholesterol from the plasma membrane in mammalian cells under normal growth conditions. This is supported by a recent study showing that RNA interference against clathrin heavy chain in hepatoma cells results in exclusive staining of the cell surface by filipin after cholesterol reloading, while no intracellular sterol could be detected using this probe (Ge et al. 2008). Despite the reasonable vesicular sterol uptake in J774 cells, only a minor fraction of DHE can be detected in endocytic vesicles. We cannot exclude that all $\mathrm{Tf}$ containing endocytic vesicles have also 

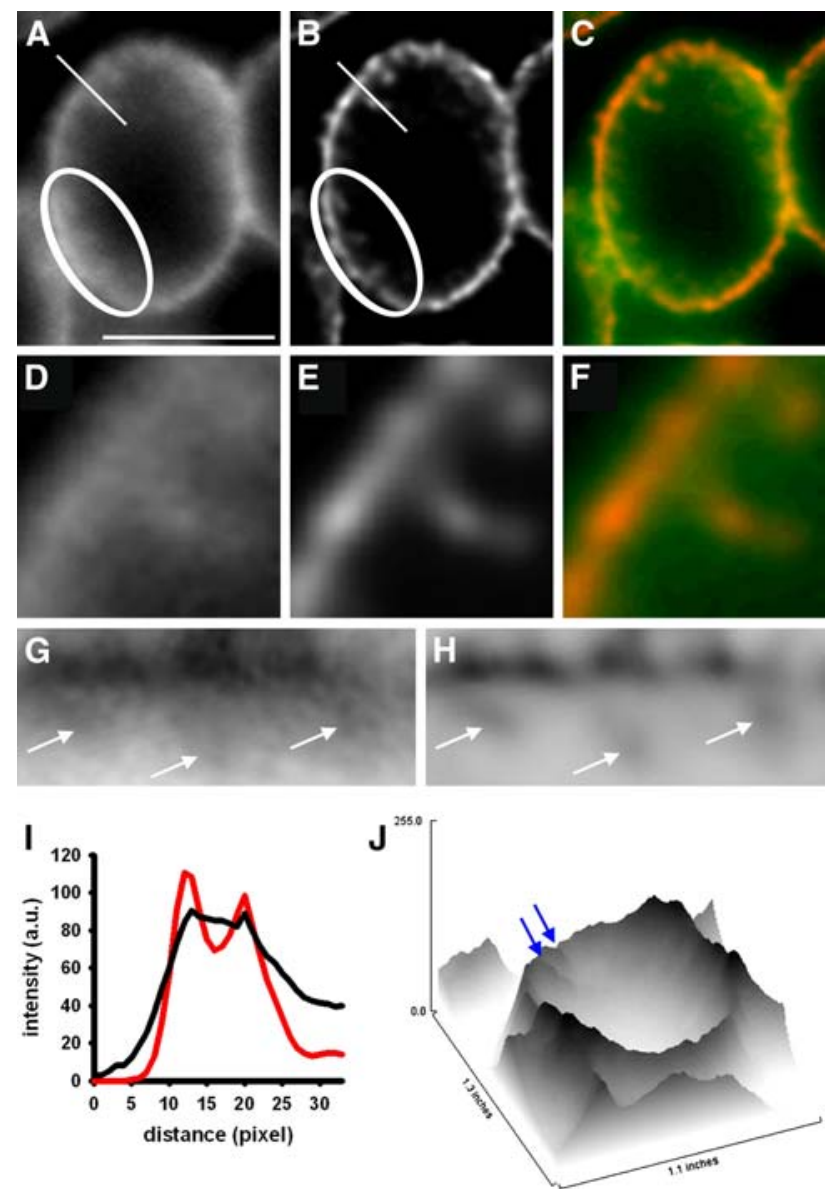

Fig. 9 Limits of detecting DHE containing vesicles in the presence of high cytoplasmic sterol staining. J774 cells were labeled for $5 \mathrm{~min}$ at $37^{\circ} \mathrm{C}$ with $\mathrm{DHE} / \mathrm{M} \beta \mathrm{CD}$, washed, chased for $3 \mathrm{~min}$ at $37^{\circ} \mathrm{C}$ and imaged on a wide field microscope with appropriate filter settings. a Raw data image. b The same image after applying a 2-dimensional deconvolution algorithm implemented in Hyugens software (SVI, Hilversum, The Netherlands). c Color overlay with raw data image in green and deconvolved image in red. $\mathbf{d}-\mathbf{f}$ Zoomed regions corresponding to area under the straight white line in $\mathbf{a}-\mathbf{c}$, respectively. $\mathbf{g}, \mathbf{h}$ Zoomed region obtained from images $\mathbf{a}, \mathbf{b}$ after straightening the area encircled by the white ellipse. $\mathbf{g}$, $\mathbf{h}$ Endocytic vesicles can be detected close to the plasma membrane (arrows). i Line scan of DHE intensity along the straight white line in $\mathbf{a}$ (black curve) and $\mathbf{b}$ (red curve). The black curve for the raw data reveals the intensity gradient of DHE from the plasma membrane towards the cell center. Endocytic vesicles create a small peak at pixel position 20. Deconvolution (red curve) reduces the cytoplasmic background and improves visibility of the DHE containing vesicle. $\mathbf{j}$ Surface plot of the raw data image shown in a created using ImageJ. Arrows indicate the position of two endocytic vesicles corresponding to the position under the white line in $\mathbf{a} . \mathbf{g}, \mathbf{h}$ Inverted images. Bar $10 \mu \mathrm{m}$

DHE in amounts to be too low for detection by our imaging system. In other words, it is possible that endocytosis of DHE in small amounts into a large number of endocytic vesicles contributes significantly to vesicular sterol uptake. In fact, calculation of intensity values of DHE in selected regions of the cell from images of $\mathbf{J} 774$ cells right after pulse-labeling with $\mathrm{DHE} / \mathrm{MCD}$ reveals that diffuse cytoplasmic DHE staining creates an almost linearly decreasing intensity gradient from the plasma membrane towards the cell center (Fig. 9). Consequently, the contrast generated by a newly formed DHE containing vesicle close to the plasma membrane is very low (i.e., DHE intensity in the vesicle as well as in the plasma membrane is only $15 \%$ above the diffuse cytoplasmic fluorescence close to the cell membrane). Giving a lower detection limit of $5 \%$ above cytoplasmic background intensity for an endocytic vesicle in the cytoplasm, one can conclude that endocytic vesicles should contain at least $90 \%$ of the mean DHE intensity in the plasma membrane to be detected as newly formed DHE positive vesicles. Thus, even a slight sorting of sterol during the endocytic process of 1:1.2 between plasma membrane and endocytic vesicle corresponding to $20 \%$ less sterol in the endoctic vesicle will abolish any detectable DHE signal in this vesicle. Thus, it is likely that the significant non-vesicular sterol uptake resulting in high diffuse cytoplasmic DHE fluorescence makes the detection of newly formed endocytic vesicles containing a little less DHE than the plasma membrane impossible. Deconvolution enhances contrast slightly and increases the ability to determine the location of endocytic vesicles with DHE; it does not allow, however, for detection of vesicles not creating any contrast in the original data (Fig. 9). Once sterol containing vesicles move away from the cell surface towards the cell center, they are easier to detect because the intensity profile of diffuse cytoplasmic sterol staining drops towards the perinuclear region. Thus, non-vesicular intracellular sterol transport limits the achievable contrast of DHE containing vesicles close to the cell surface and lowers thereby the ability of the imaging system to detect sterol endocytosis.

Clathrin-coated pits (CCP), basket like invaginations of the plasma membrane, were proposed to exclude cholesterol based on early experiments using the antibiotic sterolbinding filipin (Montesano et al. 1979). By electron microscopy (EM) these authors found less of the characteristic bumps caused by filipin binding to the plasma membrane in CCP compared to the rest of the cell surface (Montesano et al. 1979, 1981). Subsequently, it was shown that filipin does not have access to CCP due to the associated clathrin coat, removing the coat revealed the same cholesterol content in early endocytic vesicles containing LDL as found in the plasma membrane (McGookey et al. 1983). The same was found for endocytic vesicles prepared from rat liver (Steer et al. 1984). Filipin was also shown to disrupt membranes and to extract cholesterol, and is therefore not a quantitative measure of lateral membrane sterol distribution (Wüstner 2007a and Ref. therein). By assessing plasma membrane distribution of DHE during the process of sterol endocytosis we show that endocytic sites are not particularly enriched or depleted in DHE (see 
Fig. 2). These data demonstrate that the process of sterol endocytosis does not involve formation of microscopically resolvable sterol domains at the cell surface. ATP-depletion, on the other hand triggered clustering of Alexa546-Tf and DHE in shallow surface invaginations. This could be a result of inhibited clathrin-exchange in response to ATPdepletion as reported earlier using GFP-tagged clathrin (Wu et al. 2001).

Caveolae are abundant in adipocytes and endothelial cells and are classically considered as sterol enriched plasma membrane domains (Ikonen 2000; Simons and Ikonen 1997). Evidence for cholesterol enrichment in caveolae is, however, circumstantial and is either based on biochemical fractionation assays, or relies on filipin staining experiments (Ikonen 2000; Murata et al. 1995; Ortegren et al. 2004). In the latter case, the same arguments as for visualization of sterol in CCP applies; Seven and Simons (1983) showed that technical limitations led to the wrong conclusion in an earlier paper by Montesano (1979) who found, based on filipin staining of smooth muscle cell membranes, a relative cholesterol enrichment in caveolae (Montesano 1979; Severs and Simons 1983). The biochemical evidence is based on isolation of caveolae either by ultracentrifugation in carbonate buffers (Ortegren et al. 2004) or using cold non-ionic detergent: detergent resistant membrane proteins including caveolin and lipids including cholesterol have been proposed to exist in liquid ordered domains in the native plasma membrane prior to detergent treatment (Simons and Ikonen 1997). This assay, however, has been questioned in many recent biophysical and cell biological studies showing basically that detergent treatment induces domains and clusters membrane components in caveolae (Heerklotz 2002; Mayor and Maxfield 1995). Thus, even while being stated in many studies, the experimental evidence for cholesterol accumulation in caveolae is rather poor and indirect, because it relies on membrane fractionation and isolation of caveolae being prone to redistribution of membrane lipids and proteins. Here, we show for the first time in living cells that fluorescent sterols with properties closely resembling those of cholesterol do not accumulate in cell surface caveolae. Both, DHE and CTL, have a homogeneous distribution in the plasma membrane with no particular enrichment in caveolae labeled with GFP-Cav1 in CHO cells or in adipocytes. Moreover, caveolar-rich caves, a specific surface feature of adipocytes, did not contain more of the fluorescent sterols than of the lipid probe DiIC12 which prefers liquid-disordered phases in model membranes (Garvik et al. 2008). This result leads to the important conclusion that caveolae have a comparable sterol content as the rest of the plasma membrane. Note that CTL differs from cholesterol only in having two extra double bonds in the sterol ring system making it slightly fluorescent, while DHE has an extra methyl group and double bond in the side chain compared to CTL. Both analogs differ from cholesterol in having a lower ability to condense acyl-chain packing of phospholipids in model membranes (Scheidt et al. 2003). Thus, it is likely that CTL and to an even higher extent DHE have a lower potential to induce the liquid ordered phase proposed to be involved in plasma membrane domain organization. It should therefore be emphasized that we use here both sterols as marker for cholesterol and not as replacement of plasma membrane cholesterol. We showed previously that our labeling method replaces about $10 \mathrm{~mol} \%$ of cholesterol for DHE, keeping structural perturbation of the membrane at a minimum (Wüstner et al. 2002). Up to this concentration, DHE mimics cholesterol fairly well in its ability to order phospholipid acyl chains in model membranes (Scheidt et al. 2003) as well as is potential to stiffen lipid bilayers (Garvik et al. 2008). Probe partitioning measurements using fluorescence microscopy showed recently that CTL partitions preferentially into the liquid ordered phase of a ternary model system and we confirm this in an independent study for DHE (Baumgart et al. 2007; Garvik et al. 2008). Thus, the fluorescent sterols DHE and CTL are suitable as a cholesterol marker to monitor distribution of cholesterol in cellular membranes.

Using CHO cells expressing GFP-Cav1, we find evidence for internalization of some fluorescent sterol via caveolar endocytosis. It is possible that expression of caveolin triggers this sterol uptake pathway probably requiring the binding of sterol to the caveolin protein (Ikonen 2000; Murata et al. 1995). Time-lapse microscopy in combination with statistical image analysis furthermore establishes that fluctuations in staining intensity of DHE and the lipid probe DiIC12 are indistinguishable and resemble the surface dynamics of living cells. We therefore conclude that plasma membrane sterol does not phase segregate and that the characteristic properties of caveolae are not mediated by an increased sterol content of these cell surface specializations (Ikonen 2000; Maxfield and Wüstner 2002; Mukherjee and Maxfield 2004). The reported binding of cholesterol to caveolin could induce a transient stabilization of cholesterol in close vicinity of caveolin proteins (Murata et al. 1995). Binding of cholesterol to caveolin and caveolin-facilitated transport of newly synthesized cholesterol to the cell surface requires palmitoylation of the caveolin protein (Smart et al. 1996; Uittenbogaard and Smart 1998). Once arrived at the plasma membrane, this cholesterol was found to diffuse from caveolae over the whole cell surface (Smart et al. 1996). We speculate that transient interaction of sterol with caveolin slows sterol diffusion on a nanometer scale in vicinity of caveolin without apparent lateral sterol enrichment in caveolae. This slowed diffusion could result in a higher residence time of sterol in caveolae and thereby 
uptake of sterol by caveolar endocytosis. In fact, DHE has been shown to have an increased fluorescence polarization value indicating restricted mobility in proximity to caveolin in isolated membrane fractions (Gallegos et al. 2004, 2006). We find that the majority of intracellular sterol, however, does not co-localize with GFP-Cav1 supporting earlier results (Naslavsky 2004). The methods developed and applied in this study set the stage towards a future systematic exploration of proteins being involved in vesicular sterol uptake from the plasma membrane.

Acknowledgments D.W. is grateful to Dr. Frederick R. Maxfield (Weill Medical College of Cornell University, New York, USA) for fluorescence microscopy instrumentation, reagents and cell culture facility for initital experiments described in this paper (supported by NIH grant DK27083) and to Dr. Bo van Deurs, University of Kopenhagen, Denmark for generously providing the plasmid for GFPCav1. Connie Gram is acknowledged for excellent technical assistance with adipocyte cell culture and cell transfection. Anja Zeigerer (Max Planck Institute for Molecular Cell Biology and Genetics) is acknowledged for advice regarding electroporation of adipocytes. D.W. acknowledges funding by grants of the Danish Heart Association Hjerteforeningen, the Diabetes Foundation Diabetesforeningen, and D.W. and N.F. acknowledge funding by grants of the Danish Research Council.

Open Access This article is distributed under the terms of the Creative Commons Attribution Noncommercial License which permits any noncommercial use, distribution, and reproduction in any medium, provided the original author(s) and source are credited.

\section{References}

Abney JR, Meliza CD, Cutler B, Kingma M, Lochner JE, Scalettar BA (1999) Real-time imaging of the dynamics of secretory granules in growth cones. Biophys J 77:2887-2895

Aschenbrenner L, Naccache SN, Hasson T (2004) Uncoated endocytic vesicles require the unconventional myosin, Myo6, for rapid transport through actin barriers. Mol Biol Cell 15:2253-2263

Bar LK, Chong PL, Barenholz Y, Thompson TE (1989) Spontaneous transfer between phospholipid bilayers of dehydroergosterol, a fluorescent cholesterol analog. Biochim Biophys Acta 983:109_ 112

Baumann NA, Sullivan DP, Ohvo-Rekila H, Simonot C, Pottekat A, Klaassen Z, Beh CT, Menon AK (2005) Transport of newly synthesized sterol to the sterol-enriched plasma membrane occurs via nonvesicular equilibration. Biochemistry 44:5816-5826

Baumgart T, Hunt G, Farkas ER, Webb WW, Feigenson GW (2007) Fluorescence probe partitioning between Lo/Ld phases in lipid membranes. Biochim Biophys Acta 1768:2182-2194

Blatter C (2003) Wavelets - Eine Einführung. Advanced lecture notes on mathematics. Vieweg Verlag, Braunschweig/Wiesbaden Germany

Daumas F, Destainville N, Millot C, Lopez A, Dean D, Salome L (2003) Confined diffusion without fences of a G-protein-coupled receptor as revealed by single particle tracking. Biophys J 84:356-366

Dormann D, Libotte T, Weijer CJ, Bretschneider T (2002) Simultaneous quantification of cell motility and protein-membrane- association using active contours. Cell Motil Cytoskeleton $52: 221-230$

Gallegos AM, McIntosh AL, Atshaves BP, Schroeder F (2004) Structure and cholesterol domain dynamics of an enriched caveolae/raft isolate. Biochem J 382:451-461

Gallegos AM, Storey SM, Kier AB, Schroeder F, Ball JM (2006) Structure and cholesterol dynamics of caveolae/raft and nonraft plasma membrane domains. Biochemistry 45:12100-12116

Garvik O, Ipsen JH, Wüstner D (2008) Domain partitioning of the fluorescent sterol dehydroergosterol and its effect on membrane bending rigidity (submitted for publication)

Ge L, Qi W, Miao HH, Cao J, Qu YX, Li BL, Song BL (2008) The cholesterol absorption inhibitor ezetimibe acts by blocking the sterol-induced internalization of NPC1L1. Cell Metab 7:508-519

Ghosh RN, Gelman DL, Maxfield FR (1994) Quantification of lowdensity lipoprotein and transferrin endocytic sorting in HEp2 cells using confocal microscopy. J Cell Sci 107:2177-2189

Hao M, Lin SX, Karylowski OJ, Wüstner D, McGraw TE, Maxfield FR (2002) Vesicular and non-vesicular sterol transport in living cells. The endocytic recycling compartment is a major sterol storage organelle. J Biol Chem 277:609-617

Hartwig Petersen N, Faergeman NJ, Yu L, Wüstner D (2008) Kinetic imaging of NPC1L1 and sterol trafficking between plasma membrane and recycling endosomes in hepatoma cells. J Lipid Res (in press)

Heerklotz H (2002) Triton promotes domain formation in lipid raft mixtures. Biophys J 83:2693-2701

Ikonen E, Parton RG (2000) Caveolins and cellular cholesterol balance. Traffic 1:212-217

Inoue S, Spring KR (1997) Video microscopy, 2nd edn. Plenum Press, New York

Kaplan MR, Simoni RD (1985) Transport of cholesterol from the endoplasmic reticulum to the plasma membrane. J Cell Biol 101:446-453

Kobrinsky E, Mager DE, Bentil SA, Murata S, Abernethy DR, Soldatov NM (2005) Identification of plasma membrane macroand microdomains from wavelet analysis of FRET microscopy. Biophys J 88:3625-3634

Kocsis E, Trus BL, Steer CJ, Bisher ME, Steven AC (1991) Image averaging of flexible fibrous macromolecules: the clathrin triskelion has an elastic proximal segment. J Struct Biol 107:6-14

Li Y, Prinz WA (2004) ATP-binding cassette (ABC) transporters mediate nonvesicular, raft-modulated sterol movement from the plasma membrane to the endoplasmic reticulum. J Biol Chem 279:45226-45234

Lin SX, Grant B, Hirsh D, FR M (2001) Rme-1 regulates the distribution and function of the endocytic recycling compartment in mammalian cells. Nat Cell Biol 3:567-572

Mager DE, Abernethy DR (2007) Use of wavelet and fast Fourier transforms in pharmacodynamics. J Pharmacol Exp Ther 321:423-430

Maxfield FR, Wüstner D (2002) Intracellular cholesterol transport. J Clin Invest 110:891-898

Mayor S, Maxfield FR (1995) Insolubility and redistribution of GPIanchored proteins at the cell surface after detergent treatment. Mol Biol Cell 6:929-944

McGookey DJ, Fagerberg K, Anderson RG (1983) Filipin-cholesterol complexes form in uncoated vesicle membrane derived from coated vesicles during receptor-mediated endocytosis of low density lipoprotein. J Cell Biol 96:1273-1278

Montesano R (1979) Inhomogeneous distribution of filipin-sterol complexes in smooth muscle cell plasma membrane. Nature 280:328-329

Montesano R, Perrelet A, Vassalli P, Orci L (1979) Absence of filipin-sterol complexes from large coated pits on the surface of culture cells. Proc Natl Acad Sci USA 76:6391-6395 
Montesano R, Vassalli P, Orci L (1981) Structural heterogeneity of endocytic membranes in macrophages as revealed by the cholesterol probe, filipin. J Cell Sci 51:95-107

Mukherjee S, Maxfield FR (2004) Membrane domains. Annu Rev Cell Dev Biol 20:839-866

Mukherjee S, Ghosh RN, Maxfield FR (1997) Endocytosis. Physiol Rev 77:759-803

Murata M, Peränen J, Schreiner R, Wieland F, Kurzchalia TV, Simons K (1995) VIP21/caveolin is a cholesterol-binding protein. Proc Natl Acad Sci USA 92:10339-10343

Naslavsky N, Weigfert R, Donaldson JG (2004) Characterization of a non-clathrin endocytic pathway: membrane cargo and lipid requirements. Mol Biol Cell 15:3542-3552

Ortegren U, Karlsson M, Blazic N, Blomqvist M, Nystrom FH, Gustavsson J, Fredman P, Strålfors P (2004) Lipids and glycosphingolipids in caveolae and surrounding plasma membrane of primary rat adipocytes. Eur J Biochem 271:2028-2036

Parton RG, Richards AA (2003) Lipid rafts and caveolae as portals for endocytosis: new insights and common mechanisms. Traffic 4:724-738

Parton RG, Molero JC, Floetenmeyer M, Green KM, James DE (2002) Characterization of a distinct plasma membrane macrodomain in differentiated adipocytes. J Biol Chem 277:4676946778

Sage D, Neumann FR, Hediger F, Gasser SM, Unser M (2005) Automatic tracking of individual fluorescence particles: application to the study of chromosome dynamics. IEEE Trans Image Processing 14:1372-1383

Salzmann NH, Maxfield FR (1989) Fusion accessibility of endocytic compartments along the recycling and lysosomal endocytic pathways in intact cells. J Cell Biol 109:2097-2104

Saxton MJ, Jacobson K (1997) Single-particle tracking: applications to membrane dynamics. Annu Rev Biophys Biomol Struct 26:373-399

Scheidt HA, Müller P, Herrmann A, Huster D (2003) The potential of fluorescent and spin-labeled steroid analogs to mimic natural cholesterol. J Biol Chem 278:45563-45569

Severs NJ, Simons HL (1983) Failure of filipin to detect cholesterolrich domains in smooth muscle plasma membrane. Nature 303:637-638

Simons K, Ikonen E (1997) Functional rafts in cell membranes. Nature 387:569-572

Smart EJ, Ying Y, Donzell WC, Anderson RG (1996) A role for caveolin in transport of cholesterol from endoplasmic reticulum to plasma membrane. J Biol Chem 271:29427-29435

Spink CH, Yeager MD, Feigenson GW (1990) Partitioning behavior of indocarbocyanine probes between coexisting gel and fluid phases in model membranes. Biochim Biophys Acta 1023:25-33

Steer CJ, Bisher M, Blumenthal R, Steven AC (1984) Detection of membrane cholesterol by filipin in isolated rat liver coated vesicles is dependent upon removal of the clathrin coat. J Cell Biol 99:315-319

Steyer JA, Almers W (1999) Tracking single secretory granules in live chromaffin cells by evanescent-field fluorescence microscopy. Biophys J 76:2262-2271

Thomsen P, Roepstorff K, Stahlhut M, van Deurs B (2002) Caveolae are highly immobile plasma membrane microdomains, which are not involved in constitutive endocytic trafficking. Mol Biol Cell $13: 238-250$

Uittenbogaard A, Smart EJ (1998) Palmitoylation of caveolin-1 is required for cholesterol binding, chaperone complex formation, and rapid transport of cholesterol to caveolae. J Biol Chem 275:25595-25599

Weiss M, Elsner M, Kartberg F, Nilsson T (2004) Anomalous subdiffusion is a measure for cytoplasmic crowding in living cells. Biophys J 87:3518-3524

Wu X, Zhao X, Baylor L, Kaushal S, Eisenberg E, Greene LE (2001) Clathrin exchange during clathrin-mediated endocytosis. J Cell Biol 155:291-300

Wüstner D (2005) Improved visualization and quantitative analysis of fluorescent membrane sterol in polarized hepatic cells. J Microsc 220:47-64

Wüstner D (2007a) Fluorescent sterols as tools in membrane biophysics and cell biology. Chem Phys Lipids 146:1-25

Wüstner D (2007b) Plasma membrane sterol distribution resembles the surface topography of living cells. Mol Biol Cell 18:211-228

Wüstner D, Faergeman NJ (2008) Chromatic aberration correction and deconvolution for UV sensitive imaging of fluorescent sterols in cytoplasmic lipid droplets. Cytometry A 73(8):727-744

Wüstner D, Herrmann A, Hao M, Maxfield FR (2002) Rapid nonvesicular transport of sterol between the plasma membrane domains of polarized hepatic cells. J Biol Chem 277:3032530336

Wüstner D, Mondal M, Tabas I, Maxfield FR (2005) Direct observation of rapid internalization and intracellular transport of sterol by macrophage foam cells. Traffic 6:396-412

Yamashiro DJ, Tycko B, Fluss SR, Maxfield FR (1984) Segregation of transferrin to a mildly acidic ( $\mathrm{pH}$ 6.5) para-Golgi compartment in the recycling pathway. Cell 37:789-800

Zeigerer A, Lampson MA, Karylowski O, Sabatini DD, Adesnik M, Ren M, McGraw TE (2002) GLUT4 retention in adipocytes requires two intracellular insulin-regulated transport steps. Mol Biol Cell 13:2421-2435

Zha X, Pierini LM, Leopold PL, Skiba PJ, Tabas I, Maxfield FR (1998) Sphingomyelinase treatment induces ATP-independent endocytosis. J Cell Biol 140:39-47

Zhang W, McIntosh AL, Xu H, Wu D, Gruninger T, Atshaves B, Liu JC, Schroeder F (2005) Structural analysis of sterol distributions in the plasma membrane of living cells. Biochemistry 44:28642884 